\title{
Verbreitung und Ausbildung Elster-zeitlicher Ablagerungen zwischen Elm und Flechtinger Höhenzug
}

\author{
Harald Elsner*)
}

ElsNer, H. (2003): Verbreitung und Ausbildung Elsterzeitlicher Ablagerungen zwischen Elm und Flechtinger Höhenzug. - Eiszeitalter und Gegenwart, 52: 91-116; Hannover.

Keywords: Lower Saxony, Saxony-Anhalt, Schöningen lignite pit, Morsleben valley, Elsterian ice age, Elsterian interstadials, Elsterian stratigraphy, Elsterian oscillations, Elsterian tills.

\section{Widmung}

Diese Veröffentlichung ist Herrn Dipl.-Geol. Hans ZierMANN (4.9.1934-20.3.2000), Berlin, gewidmet, der den Projektteil Quartär bei den „Deckgebirgsuntersuchungen ERA Morsleben“" seitens der Bundesanstalt für Geowissenschaften und Rohstoffe (BGR) leitete. Aufgrund seiner hervorragenden Kenntnis des nordostdeutschen Quartärs wird Hans Ziermann allen, die ihn kannten, als erfahrener und stets freundlicher Diskussionspartner in Erinnerung bleiben. Ohne seine Unterstützung wäre diese Arbeit nicht möglich gewesen.

Kurzfassung: Im östlichen Niedersachsen und westlichen Sachsen-Anhalt wurden in den letzten Jahrzehnten in zwei großen Untersuchungskampagnen mächtige quartäre Ablagerungen beobachtet und dokumentiert. Die Elster-zeitlichen Anteile dieser Ablagerungen sind Gegenstand dieser Veröffentlichung. Ablagerungen der ältesten Vereisung aus diesem Gebiet wurden schon vor über 100 Jahren beschrieben. Im Helmstedter Braunkohlerevier waren über lange Zeit über mächtigen VorschüttSchmelzwassersanden zwei Elster-Grundmoränen aufgeschlossen. Stillwasserbildungen verschiedenster Ausbildung waren Zeugen des periglaziären Geschehens in diesem Raum. Am Ende des Pleniglazials kamen in kleinen Seen limnische Sedimente zur Ablagerung, die Hinweise auf mehrere Spätelster-Interstadiale lieferten.

Im Zuge einer sehr umfangreichen ingenieurgeologischen Erkundung im nur wenige Kilometer entfernten Allertal bei Morsleben wurden dort einige Jahre später zahlreiche Kernbohrungen niedergebracht und intensiv geologisch

*) Anschrift des Verfassers: Dr. Harald Elsner, EurGeol, Salbeiweg 1A, D-38239 Salzgitter dokumentiert und beprobt. Die erbohrten Ablagerungen ähneln den Elster-zeitlichen Sedimenten aus dem Tagebau Schöningen, sind jedoch durch zahlreiche Subrosionssenken noch mächtiger. Hierdurch lassen sich auch mehrere kleine Oszillationen zu Beginn der Überfahrung durch das Elster-Inlandeis nachweisen. An einer Stelle im Allertal ist zudem relativ sicher eine jüngste dritte Elster-Grundmoräne nachweisbar. Die periglazären Bildungen kamen hier in einem eher fluviatil geprägten Milieu zur Ablagerung, das auch die Erhaltung sicher zuordnenbarer Spätelster-Interstadiale behinderte. Eine Korrelation der beobachteten Ablagerungen mit denen anderer Räume ist bisher nur regional, nicht jedoch überregional möglich.

[Distribution and form of deposits of Elsterian age between Elm and Flechtingen ridge]

Abstract: In one eastern area of Lower Saxony, and in one western area of neighbouring Saxony-Anhalt thick deposits of Quaternary age were observed during two extensive exploration campaigns within the last decades. The strata of Elsterian age within these deposits shall be described within this report. Deposits of this oldest glaciation in this region were already documented more than 100 years ago. In the Helmstedt lignite mining area two tills of Elsterian age above glaciofluvial sands had been observable for many years. Various fine-size sediments were deposited in the periglacial environment. Limnic sediments still containing pollen of late Elsterian interstadials were deposited in small ponds, too.

A few years later in the neighbouring Aller valley near Morsleben many cores taken and described during an extensive exploration campaign showed similar strata as in the Helmstedt lignite mining area. Due to salt leaching in rocks underneath, however, those strata were even thicker allowing proof of small oscillations before complete Elsterian ice shield coverage. In one region of the Aller valley even a third uppermost till of Elsterian age can be found. Deposition of periglacial sediments in this valley were dominated by a fluvial regime, which also prohibited the preservation of clearly classifiable interstadial deposits. A correlation of all these deposits of Elsterian age is only possible within the region but not over far distances. 


\section{Einleitung}

In den letzten 15 Jahren sind in dem Gebiet zwischen den Mittelgebirgen des Elms (östliches Niedersachsen) und des Flechtinger Höhenzuges (westliches Sachsen-Anhalt) umfangreiche quartärgeologische Arbeiten durchgeführt worden, die wichtige Rückschlüsse auf das Klimageschehen während des Mittel- und Jungpleistozäns zulassen. Im Gegensatz zu den Ergebnissen aus den interglazialen Ablagerungen des Tagebaus Schöningen südlich Helmstedt, die bereits internationale Beachtung fanden (z. B. Tagesexkursion B 4 während der INQUA 1995), blieben die Beobachtungen zum glaziären Geschehen aus dem o. g. Raum weitgehend unbeachtet. Ein Grund hierfür mag die Darstellung der Ergebnisse fast ausschließlich in Form von unveröffentlichten Studien und Diplomarbeiten, letztere am Institut für Geologie und Paläontologie der Universität Hannover, gewesen sein. Im Zeitraum 1994/95 erzielte der Autor in Zusammenarbeit mit Kollegen neue Erkenntnisse im Rahmen eines wissenschaftlichen Programms zur Erkundung des Deckgebirges im Bereich des Endlagers für radioaktive Abfälle Morsleben (ERAM), nur wenige Kilometer vom Tagebau Schöningen entfernt. Hierdurch war es möglich, weitere pleistozäne Schichtenabfolgen zu dokumentieren sowie neue und ältere Beobachtungen stratigraphisch zu korrelieren. Als Ergebnis liegen nun umfangreiche Erkenntnisse aus einem Raum vor, der als Bindeglied zwischen Gebieten mit mächtigem Quartär (Norddeutsches Tiefland) und dem Mittelgebirgsraum sowie seiner südlichen Fortsetzung (Sachsen) mit seinen zum Teil zahlreichen Erosionslücken steht. Besonders die Erkenntnisse zum Geschehen während der Elster-Eiszeit sind von überregionaler Bedeutung und sollen hier dargestellt werden.

\section{Geologisch-topographisch-hydrologischer Überblick}

Das Gebiet zwischen Elm und Flechtinger Höhenzug umfaßt den nordöstlichen Teil des subherzynen Beckens, das durch eine komplexe Abfolge von herzynisch streichenden Strukturen gekennzeichnet ist. Nordöstlich des Muschelkalksattels des Elms (höchste Erhebung: Kuxberg, 321,9 m ü. NN) folgt der Helmstedt-Staßfurter Sattel mit einer NW-SEErstreckung von $80 \mathrm{~km}$. Zu beiden Seiten dieses Sattels aus Zechsteinsalzen sind Mulden ausgebildet (GOK ca. 95-140 m ü. NN), in denen mächtige tertiäre und quartäre Sedimente zur Ablagerung kamen. Eozäne, z. T. auch oberpaläozäne Braunkohleflöze in diesen Mulden stehen seit über 200 Jahren im Abbau (gegenwärtig noch im Tagebau Schöningen). An die Ostmulde bei Helmstedt schließt sich nordöstlich der Lappwald an (höchste Erhebung: Walbecker Warte, 194,3 m ü. NN), der an seiner Oberfläche aus Gesteinen des Lias und des Rhät aufgebaut ist.

Im nordöstlich gelegenen Allertal stehen zwischen Ummendorf und Weferlingen (GOK ca. 90-130 m ü. NN) unter teils mächtigen quartären und tertiären/oberkretazischen Sedimenten verbreitet Triasund Jura-Gesteine an. Diese überdecken einen weiteren NW-SE-streichenden Zechsteinsalzsattel, aus dem früher durch mehrere Schächte v. a. Kalisalz gewonnen wurde. Bei Morsleben wird ein Teil der alten Abbauhohlräume seit einigen Jahren zur Einlagerung von Abfällen genutzt. Nordöstlich an die Allertalstruktur leitet die Weferlinger-Schönebecker Triasplatte (GOK ca. 110-160 m ü. NN) zum Paläozoikum-Aufbruch des Flechtinger Höhenzuges über, der am Hasenberg mit 152,0 m ü. NN seine höchste Erhebung findet.

Zusammenfassend betrachtet ergibt sich im Untersuchungsgebiet das Bild dreier NW-SE-streichender Höhenrücken (Elm, Lappwald, Flechtinger Höhenzug), die durch morphologische Einsenkungen (Helmstedt-Staßfurter Sattel mit Randsenken, Allertalstruktur) getrennt sind. Die verschiedenen Höhenzüge stellen Wasserscheiden dar, so daß die Entwässerung im NE über die Aller, im NW über die Schunter Richtung Oker bzw. im $S$ über die Mißaue, die Schöninger Aue, den Großen Graben und die Bode in die Saale erfolgt. Einen Überblick über die topographischen Verhältnisse gibt Abbildung 1 .

\section{Beobachtungen bis 1983}

In einer grundsätzlichen Abhandlung zur Frage der Anzahl der Vereisungen im westlich der Elbe gelegenen Raum erwähnte SchMierer (1913) Ergebnisse einer Trockenbohrung in einem Subrosionskessel bei Ummendorf. Unter Ablagerungen 


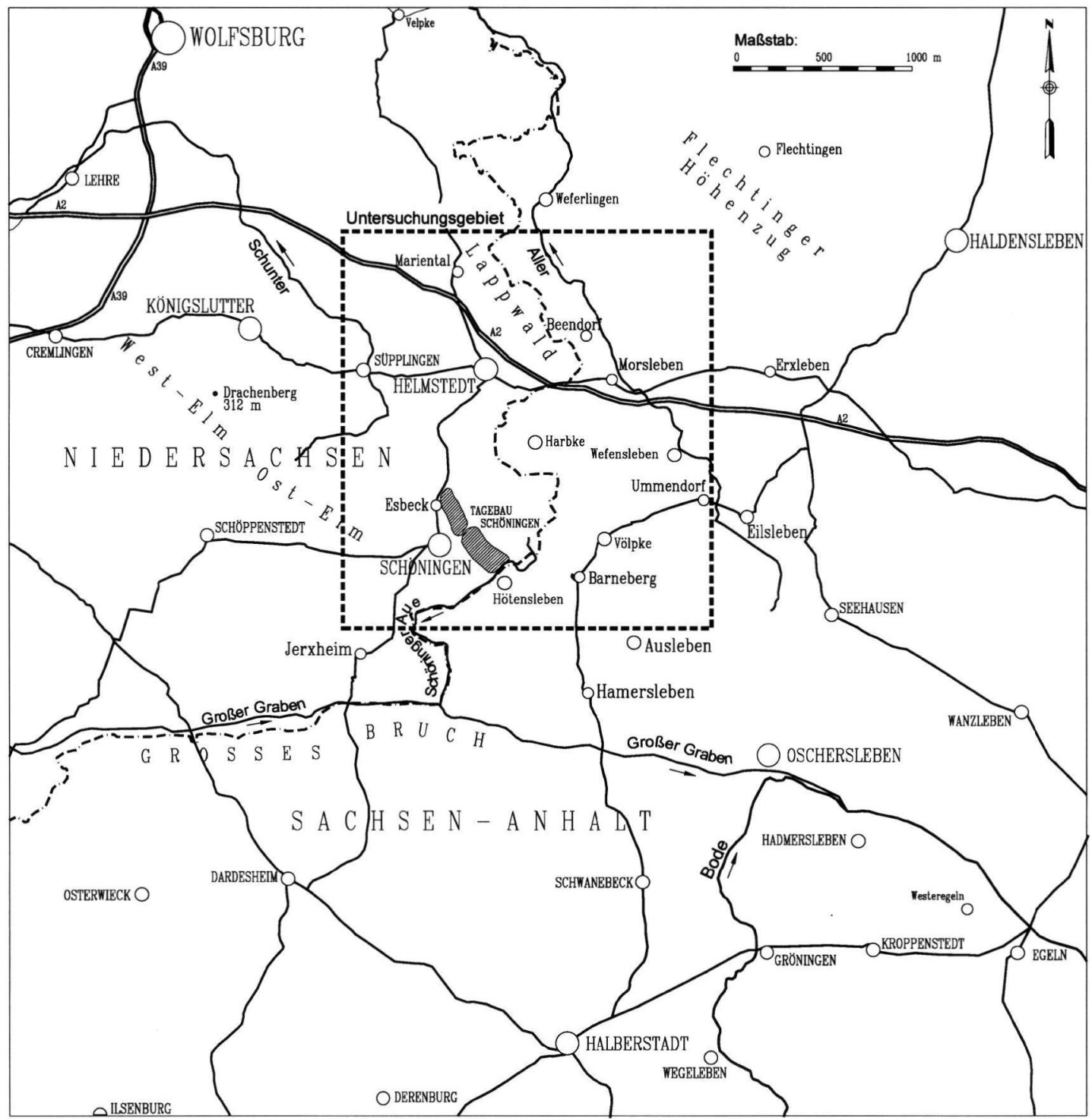

Abb. 1: Topographische Übersichtskarte des Untersuchungsraumes mit Lage des Untersuchungsgebietes.

Fig. 1: Map showing the area and site of investigation.

des Holozäns, der Saale-Kaltzeit und der HolsteinWarmzeit traten dort ab $38,0 \mathrm{~m}$ u. GOK ein "grauer, typischer Geschiebemergel" in 10,2 m Mächtigkeit, darunter 3,2 m mächtige kalkhaltige kiesige Sande, darunter in 7,6 m Mächtigkeit ein weiterer grauer Geschiebemergel und in dessen Liegendem mit 1,9 m Mächtigkeit bis zur Endteufe ein sandiger, schwach toniger, kalkhaltiger Kies mit Geröllen aus einheimischem Material an der Basis auf. Letzterer Kies wurde von SCHMierer (1913) als ausgewaschener Geschiebemergel gedeutet.
WIEGERS (1932) hob erstmals die weite Verbreitung von Ablagerungen der „I. Vereisung“, d. h. der Elster-Eiszeit, im südöstlichen Untersuchungsgebiet hervor. Ausgangspunkt seiner Beobachtungen war eine Forschungsbohrung der Preußischen Geologischen Landesanstalt, die 1925 neben einer verfallenen Ziegeleigrube bei Oschersleben abgeteuft wurde, aus der bereits SCHMIERER (1913) fossilführende interglaziale Tone beschrieben hatte. Diese Bohrung erbrachte unter einer geringmächtigen, gelblichgrauen Saale-Grundmoräne und 4,5 m 
mächtigen interglazialen „Tonmergeln“ 1,3 m mächtige, fein- und grobkörnige Schmelzwassersande sowie darunter mit über $36 \mathrm{~m}$ Mächtigkeit eine Wechselfolge von grauen Geschiebemergelund Geschiebesandbänken. In deren Liegenden folgten mit über $5 \mathrm{~m}$ Mächtigkeit hellgraue lignitreiche Schmelzwasserablagerungen bzw. bis zum anstehenden Gipskeuper eine fast $4 \mathrm{~m}$ mächtige Lokalmoräne aus nordischen und einheimischen Geschieben, Geschiebemergelbrocken und sandigem, steinigem Kies.

Ebenfalls Elster-zeitliche Ablagerungen in Form von bis $33 \mathrm{~m}$ mächtigen, horizontal- bis schwach kreuzgeschichteten, nach $S$ bzw. SW einfallenden kies- und geschiebereichen Sanden beschrieb WiEgERs (1932) von zahlreichen Stellen im Raum Hadmersleben-Wanzleben-Westeregeln. Überlagert wurden diese "Schmelzwassersande“ mit vereinzelten Auswaschungsblockpackungen durch eindeutige Ablagerungen der Bode-Hauptterrasse, in derem Hangenden wiederum zum Teil eine SaaleGrundmoräne nachgewiesen werden konnte.

Im Jahre 1960 hatte RicHTER (1961) Gelegenheit, die bereits von WAHNSCHAFFE (1880) aus den RhätSandsteinbrüchen bei Velpke erwähnten Gletscherschrammen näher zu untersuchen. Er konnte hierbei ein älteres Gletscherschrammen- und Parabelrißsystem (25 $5^{\circ}$ Richtung) von jüngeren Gletscherschrammen $\left(80^{\circ}-85^{\circ}\right.$-Richtung) unterscheiden. An einer Stelle überlagerte eine $4-5 \mathrm{~m}$ mächtige, von oben her bereits zum Teil entkalkte Grundmoräne diesen Rhät-Sandstein direkt. Geschiebezählungen belegten eindeutig eine typisch Elster-zeitliche Geschiebegemeinschaft (u. a. Quotient Flint/Nordisches Kristallin $(\mathrm{F} / \mathrm{NK})=$ 0,27-0,33). Weiter im Hangenden auftretende Ablagerungen konnten dem Drenthe-Stadium der Saale-Kaltzeit zugeordnet werden. Von RICHTER in der o. g. Grundmoräne durchgeführte Einregelungsmessungen ergaben von unten nach oben im fast schwarzen, kalkhaltigen Geschiebemergel ein Maximum der Streichrichtung von $\pm 30^{\circ}$, im mittleren, grünstichig-schwarzgrauen \pm entkalkten Geschiebemergel von $65^{\circ}$ und im obersten, ockerbraunen Geschiebelehm von $85^{\circ}$. Damit konnte Richter (1961: 128) für diesen Raum nachweisen, $\mathrm{daß}$ „sowohl hinsichtlich Einregelung der Ge- schiebe wie der Heimatgebiete der nordischen Geschiebe,...die elstereiszeitlichen Gletscher zunächst von NNE kamen und dann allmählich über NE bis fast E-W abdrehten." Später gab Richter (1962) das Theoretische Geschiebezentrum (TGZ) (zur Methodik s. Lüttig 1958 und Hoffmann \& MeYer 1997) dieser Grundmoräne mit $\lambda=15,0^{\circ}$ und $\varphi=58,61^{\circ}$ an (s. Abb. 6).

Eine Neubeprobung und Auszählung durch Prof. K.-D. Meyer (in: Merkt 1996: 42) ergab ein TGZ von $\lambda=14,43^{\circ}$ und $\varphi=58,04^{\circ}$ (s. Abb. 6).

Ende der 60er Jahre legte Look (1968) seine Untersuchungsergebnisse zum Quartär am Elm vor. Auf dem Drachenberg (312,2 m ü. NN) im WestElm wies er einer Steinsohle und einem geringmächtigen Geschiebelehmrest ein Elster-zeitliches Alter zu. Nach Auszählung von 2682 Geschieben der Grobkiesfraktion ergab sich ein Verhältnis von $5 \%$ nordischem Kristallin, $31 \%$ nordischen Sedimentgeschieben und $64 \%$ einheimischen Komponenten. Der ostfennoskandisch beeinflußten Geschiebegemeinschaft konnte ein TGZ von $\lambda=14,5^{\circ}$ und $\varphi=58,9^{\circ}$ (s. Abb. 6) sowie ein F/NK-Quotient von 1,0 zugeordnet werden.

Die bisher ausführlichste Beschreibung der quartären Verhältnisse im südlichen Teil des HelmstedtStaßfurter Salzsattels bzw. seiner Randsenken geht auf WAGENBRETH (1970) zurück. Basierend auf Bohrungen der Braunkohleerkundung konnte er drei Fazien der Elster-Grundmoränen nachweisen. Demnach tritt in diesem Raum basal häufig eine bis über $60 \mathrm{~m}$ mächtige Lokalmoräne auf, die teils aus tertiären Sanden mit Geschieben, eingekneteten Tonen, Xyliten und Geschiebemergel, teils aus einem groben Gemenge von nordischen Geschieben, einheimischen Geröllen und Tonsteinen besteht. Zu einer Art Lokalmoräne zählte WAGENBRETH auch die von Wiegers (1932) beschriebenen, bis $33 \mathrm{~m}$ mächtigen kiesigen Sande. Eine zweite, seltene, graue bis grüngraue, tonige $\mathrm{Va}$ rietät der Grundmoräne erreicht nur bis $5 \mathrm{~m}$ Mächtigkeit. Ihre Farbe wurde von WAGENBRETH durch Aufnahme von Septarienton und allochthonem „Oligozän“ erklärt. In seiner dritten und häufigsten Ausbildung ist der Geschiebemergel sandig-tonig aufgebaut und von braungrauer bis dunkelbrauner Farbe. Er erreicht Mächtigkeiten 
von ebenfalls $60 \mathrm{~m}$. Auswaschungen von Moränenmaterial (Blockpackungen, Geröllhorizonte) oder auch Sandlagen erreichen bis 2,5 m Mächtigkeit und treten an der Basis, im Hangenden, aber auch innerhalb der Grundmoräne auf. Graue bis grüngraue, feingeschichtete, von vielen kleinen Störungen durchzogene Bändertone finden sich bis maximal 5 m Mächtigkeit in verschiedenen Niveaus am Top der Lokalmoräne oder mit Schmelzwassersanden verknüpft zwischen Grundmoränenbänken. Auch in den hangenden Schichten der BodeHauptterrasse tritt unter der eigentlichen gelblichbraunen sandigen Saale-Grundmoräne eine weitere, bis $24 \mathrm{~m}$ mächtige braungraue Grundmoräne auf, die WAGEnBreth (1970) allerdings auch bereits in die Saale-Eiszeit stellte.

Zumindest eine Zweiteilung der Elster-Grundmoräne in diesem Raum ist jedoch gesichert bei einer maximalen Mächtigkeit der Elster-zeitlichen Ablagerungen in flachen Wannen und Rinnen von über $135 \mathrm{~m}$. Als Beispiel sei die Bohrung 08 (WAGENBRETH 1970) bei Oschersleben zitiert, in der unter ca. $4 \mathrm{~m}$ braungrauer Elster-Grundmoräne, 0,2 m Bänderton, dann $8 \mathrm{~m}$ Schmelzwassersande, darunter erneut $60 \mathrm{~m}$ braungraue Elster-Grundmoräne und direkt im Liegenden ca. $64 \mathrm{~m}$ Lokalmoräne erbohrt wurden.

\section{Tagebau Schöningen südlich Helmstedt}

Im Jahre 1978 wurde von der Braunschweigischen Kohlen-Bergwerke (BKB) AG, Helmstedt, mit den Aufschlußarbeiten für den Tagebau Schöningen begonnen. In zwei Baufeldern - Nord und Süd - werden seitdem Flöze der Süpplingen- und Schöningen-Formationen (früher sog. Liegende Flözgruppe) des Oberpaläozän und v. a. Untereozän (Lietzow \& RitzKowski 1996) durch Schaufelradbagger gewonnen und im Kraftwerk Buschhaus zur Stromgewinnung genutzt. Seit dem Frühjahr 1983 erfolgt eine archäologisch-quartärgeologische $\mathrm{Be}$ gleitung dieser Gewinnungsarbeiten im Rahmen des Projektes „Archäologische Schwerpunktuntersuchungen im Helmstedter Braunkohlenrevier“ unter Leitung des Institutes für Denkmalpflege, Hannover.

Im Zeitraum Frühjahr 1986 bis Herbst 1990 beteiligte sich das Institut für Geologie und Paläontologie der Universität Hannover durch Vergabe von vier Diplomarbeiten (ELSNER 1987, HARTMANN 1988, LENHART 1989, TSCHEE 1991) an diesen Untersuchungen. Hierbei wurden unter Leitung von Dr. J.-P. GROETZner vom o. g. geologischen Institut wichtige Erkenntnisse zur quartären Entwicklung dieses Raumes gewonnen. Die inzwischen mehr als 1000 Bohrungen der BKB im Tagebaugebiet lassen zudem Rückschlüsse auf Verhältnisse in abbaubedingt nicht aufgeschlossenen Feldbereichen zu. Eine Zusammenfassung und Korrelation der in verschiedenen Abbauschnitten gewonnenen, zwischenzeitlich durch den fortschreitenden Abbau fast vollständig zerstörten, besonders umfangreichen Daten zum Geschehen während der Elster-Kaltzeit wird im folgenden gegeben (vgl. Abb. 2).

\subsection{Elster-Pleniglazial}

Vor Annäherung des Elster-Inlandeises herrschten in Norddeutschland, wie auch im Helmstedter Gebiet, schon seit längerer Zeit periglaziäre Klimaverhältnisse. Durch Permafrost waren die Flöze bis in große Tiefen gefroren und die tertiären Sandschichten durch Poreneis zementiert. Mit Vorrücken des Eises und der damit einhergehenden Zufuhr gewaltiger Schmelzwassermengen taute dieser Sand oberflächennah auf und wurde einschließlich seines spezifischen Geröllinventars (Milchquarze, Quarzite, Lydite, Pyrit-/Markasitund Phosphoritkonkretionen) erodiert. Zahlreiche umstrudelte Flözklippen und Feinsandsteilwände, feinverteilte Lignitflitter im Gegensatz zu größeren Braunkohlebröckchen, vorherrschende Horizontalschichtung sowie kiesiger Grobsand in den basalen 4 bis $5 \mathrm{~m}$ der abgelagerten Schmelzwassersedimente zeugten von hohen Strömungsgeschwindigkeiten und konstanten Sedimentationsverhältnissen (Hartmann 1988, Lenhart 1989).

Tschee (1991) konnte 2 m über der Quartärbasis innerhalb dieser groben Schmelzwassersande eine bereits von HARTMANN (1988) und LENHART (1989) beobachtete Geröllpackung horizontal bis in eine Grundmoräne verfolgen. Diese älteste, maximal 3 m mächtige und nur über 150 m Längsausdehnung verfolgbare Grundmoräne war extrem schluff- und tonreich, kies- und steinarm, blockfrei sowie von dunkelgrüner bis schwarzer Farbe. Der Kalkgehalt war durchweg hoch, die obersten $0,5 \mathrm{~m}$ jedoch kalkfrei und kryoturbat beeinflußt. Das 
TAGEBAU SCHÖNINGEN

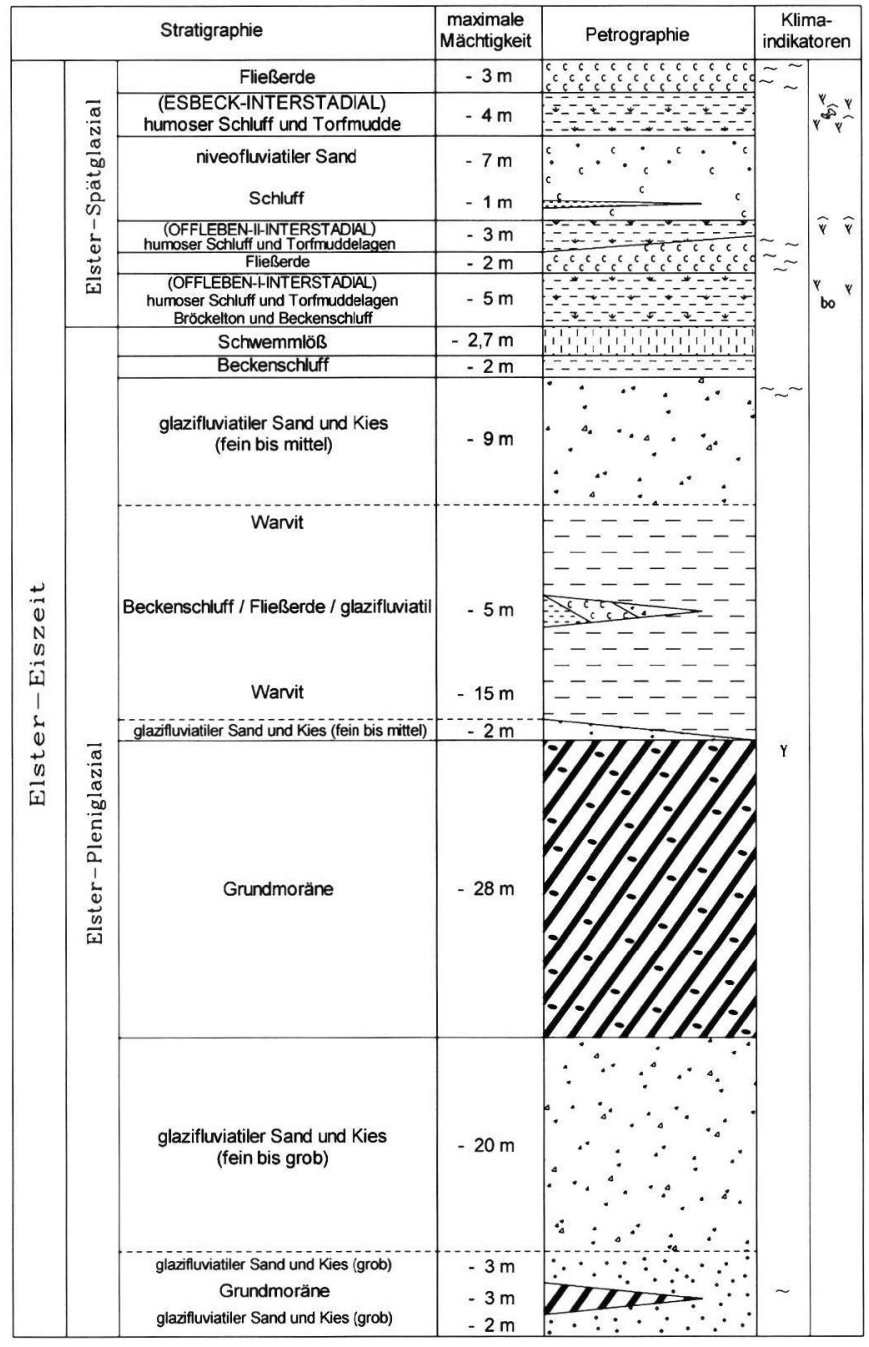

$\sim=$ Kryoturbation

= Eiskeispseudomorphosen
ALLERTAL

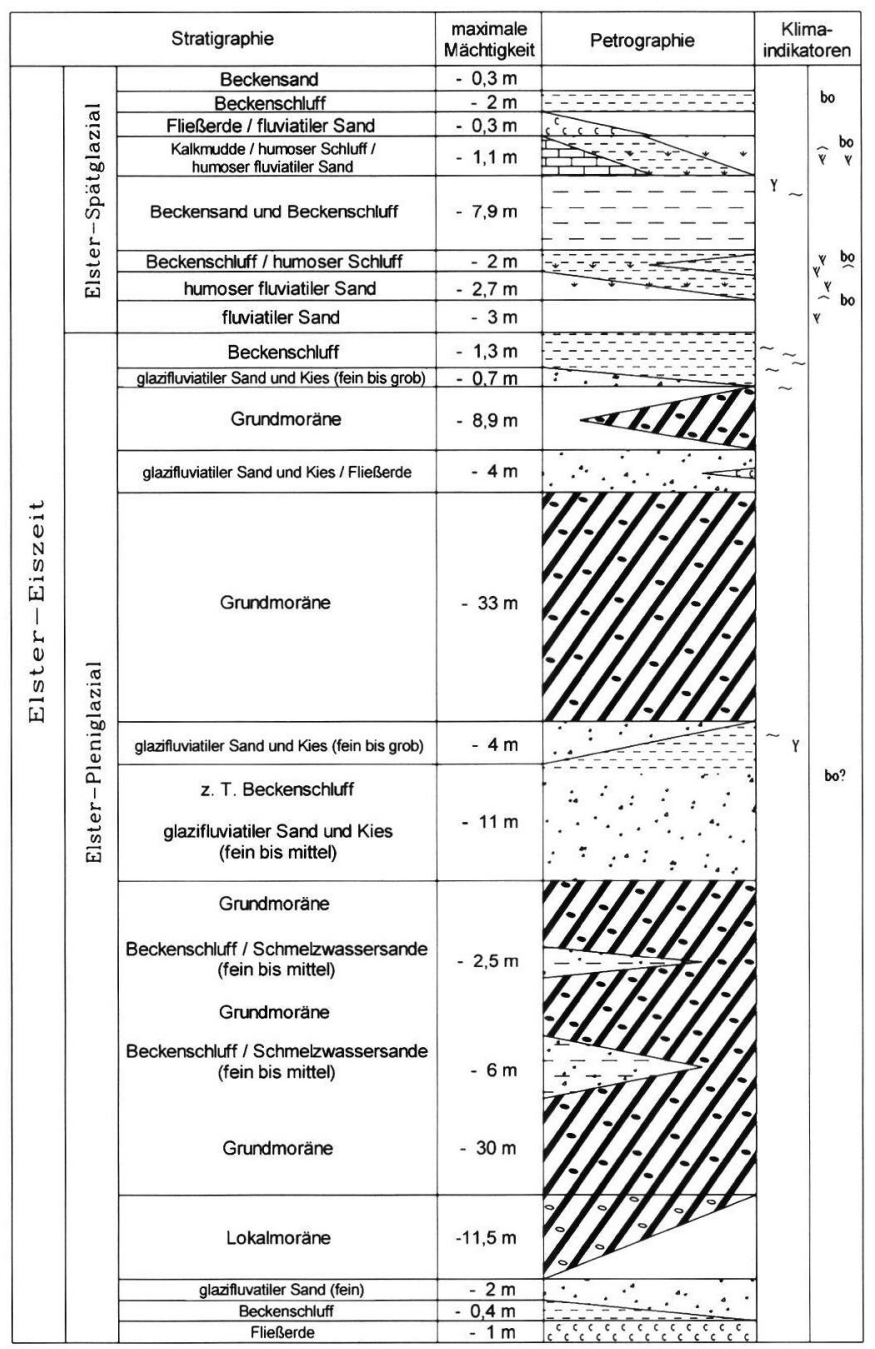

Pflanzenreste 
Verhältnis von nordischen $(\mathrm{N})$ zu einheimischmesozoischen $(\mathrm{M})$ zu südlich-paläozoischen $(\mathrm{P})$ Gesteinen (N:M:P) bzw. der F/NK-Quotient der Mittelkiesfraktion betrug 64:29:8 bzw. 0,38. Einregelungsmessungen im kryoturbat unbeeinflußten Bereich ergaben undeutliche E-W-Maxima. Eine von Prof. K.-D. Meyer (NLfB, Hannover) durchgeführte TGZ-Untersuchung von Grobkiesen ergab einen Wert von $\lambda=15,23^{\circ}$ und $\varphi=57,59^{\circ}$ (s. Abb. 5a und 6).

Der sehr grobe Schmelzwassersand (BARTHOLOMÄUS \& ELSNER 1995), in dem abbaubedingt nur über einen begrenzten Zeitraum die o. g. unterste Elster-Grundmoräne beobachtet werden konnte, ging nach oben bei gleichbleibender weißgrauer Färbung in Mittelsand mit geringen Fein- und Grobsand- sowie Kiesanteilen über. Charakteristisch waren in diesem bis über $20 \mathrm{~m}$ mächtigen hangenden Sandabschnitt außerdem lagenweise angereicherte Braunkohlenstücke und fein verteilte Lignitflitter. Schlufflagen waren stark untergeordnet vertreten; Kiesanreicherungen waren an den Basen der jeweiligen Schichtungslamellen zu finden. Vorherrschend war eine ruhige, deutliche Kreuzschichtung, die aber mit einer weiten Paralleloder einer großflächigen Schrägschichtung wechsellagerte. Häufig waren diese Schichtungseinheiten erosiv gekappt und hatten dadurch bei vergleichsweise großer Längsausdehnung (bis $50 \mathrm{~m}$ ) nur geringe Mächtigkeiten (bis $2 \mathrm{~m}$ ). Zusammen mit anderen Merkmalen konnte HaRTMANN (1988) daraus eine insgesamt sehr hohe, gleichzeitig aber ruhige und gleichmäßige Strömungsgeschwindigkeit rekonstruieren. Lokal auftretende Auf- und Abschiebungen mit Versatzbeträgen im cm- bis dmBereich konnten durch post- und synsedimentäre Sackungsvorgänge erklärt werden. Erosiv gekappte liegende Falten bis knapp über einen Meter Größe gingen dagegen auf synsedimentäre Rutschungen zurück. Der Karbonatgehalt war durch aggressive

\footnotetext{
Abb. 2: Gliederung der Elster-zeitlichen Schichtenabfolgen im Tagebau Schöningen und im Allertal (unmaßstäblich).

Fig. 2: Correlation of strata of Elsterian age in the opencast mine Schöningen and in the Aller valley (without scale).
}

Wässer der angrenzenden Tertiärschichten (diagenetisch) und Abrieb der Kalkkomponenten durch die schnell fließenden Wässer auf den Rinnensohlen (synsedimentär) stark verringert. Die Geröllzusammensetzung (Mittelkiesfraktion, kalksteinfrei gerechnet) war relativ homogen mit nordischen Anteilen von 63 bis $75 \%$, bei geringer Beteiligung von Flinten (F/NK-Quotient = 0,18-0,68\%). Der Gehalt an Milchquarzen war sehr hoch (24-36 \%).

Die erneute Annäherung des Elster-Inlandeises war durch das Auftreten von dunklen, schluffigen Lagen (Gletschertrübe) am Top dieser Vorschütt-Schmelzwassersedimente dokumentiert. Durch Einschaltung von Kies in den Schlufflagen konnte die eigentliche Grundmoräne unterschieden werden. Innerhalb mehrerer Dezimeter vertikaler Erstrekkung war diese obere Grundmoräne immer noch sehr stark sandig ausgebildet und führte einzelne grauweiße Sandlagen. In den untersten 3 bis $4 \mathrm{~m}$ des Geschiebemergels fanden sich Fetzen aus Schmelzwassersand; im Liegenden der Grundmoräne traten zudem glazitektonische Schleppfalten und Scherklüfte auf. Damit steht in Anlehnung an VAN DER MEER (1994) fest, daß zumindest der basale Teil auch dieser Grundmoräne eher durch subglaziäre tektonische Ereignisse als durch sedimentäre Prozesse beeinflußt war.

Von ElSNER (1987) konnte diese homogen und massig wirkende, schwarze bis schwarzgraue obere Elster-Grundmoräne bei einer aufgeschlossenen Mächtigkeit von maximal 13,6 m (durchschnittliche Mächtigkeit 9 bis $10 \mathrm{~m}$, in einer Bohrung wurde der gleiche Geschiebemergel bei einer Mächtigkeit von $28 \mathrm{~m}$ noch nicht durchteuft) näher untersucht werden. Dabei zeigte sich von unten nach oben eine Änderung der Matrixhauptkomponente von Feinsand zu Schluff. Geschiebe bis in Blockgröße waren häufig, Auswaschungszonen bzw. Schmelzwassersandlinsen dagegen sehr selten zu beobachten. An der Basis machten Geröllquarz und verwitterte Markasit-Konkretionen den Hauptkiesanteil aus, während sie im Dachbereich kaum noch nachweisbar waren. Generell lag der nordische Anteil in der Mittelkiesfraktion bei nur 64 bis 74 \% (vgl. Amler \& Elsner 1988, BartholomäUs 1987, BartholomäUs \& TsCheE 1993, BARTHOLOMÄUS \& ELSNER 1995), wobei der Anteil an paläozoischen Kalksteinen relativ hoch, der Anteil an Flint dagegen gering war (F/NK- 
Quotient: 0,17-0,61). Der Einfluß der Exaration im gesamten mesozoischen Vorland war sehr stark, was durch die Menge an aufgenommenen einheimischen Komponenten belegt werden konnte. Besonders Rhät-Sandsteine (Lappwald) und Muschelkalk-Gesteine (Elm, Weferlinger Triasplatte) waren sehr häufig vertreten. $\mathrm{Zu}$ nennen sind weiterhin Sandsteine des Buntsandstein (Weferlinger Triasplatte) sowie Rotliegend-Porphyre aus dem Flechtinger Höhenzug (HarTManN 1988).

Neun in verschiedenen Höhen des Elster-Geschiebemergels von ELSNER (1987) durchgeführte Einregelungsmessungen zeigen deutlich ein Schwenken der Gletscherstromrichtung von NWSE über N-S und dann NE-SW bis hin zu E-W. Dieses Bild konnte durch weitere Messungen von Hartmann (1988, n=2), LenHART $(1989, \mathrm{n}=5)$ und Tschee (1991, $\mathrm{n}=5)$ in geringermächtig aufgeschlossenem Geschiebemergel (Erosion des Dachbereiches) generell bestätigt werden. Als Nebenergebnisse der Messungen konnten im basalen Teil der Grundmoräne häufig intramoränale Störungszonen sowie im jeweiligen Dachbereich eine Überprägung durch periglaziäre Prozesse (teilweise feine Polygonrisse bis $1 \mathrm{~m}$ Tiefe) nachgewiesen werden. Nur im untersten Teil des Geschiebemergels traten zudem Geschiebe von dänischem Faxekalkstein auf, was ebenfalls für einen zuerst westfennoskandischen Eisstrom spricht (ELSNER 1987). Nach einer von Prof. K.-D. Meyer (NLfB, Hannover) durchgeführten TGZ-Untersuchung an Grobkiesen, die aus den basalen $4 \mathrm{~m}$ der Grundmoräne stammten, hatte sich hier jedoch bereits der ostfennoskandische Einfluß, nachgewiesen durch das vermehrte Auftreten von Åland-Gesteinen, deutlich durchgesetzt $\left(\lambda=15,20^{\circ}\right.$ und $\varphi=58,51^{\circ}$, s. Abb. $5 b$ und 6). Von Hartmann (1988) und Lenhart (1989) liegen weitere TGZ-Ergebnisse vor, die jedoch wegen Fehlern bei der Probenentnahme nicht repräsentativ sind. Eine von TscheE (1991) durchgeführte Aufsammlung erbrachte nach Untersuchung von Prof. K.-D. Meyer ein TGZ von $\lambda=14,85^{\circ}$ bzw. $\varphi=58,35^{\circ}$ (s. Abb. 5 c und 6).

Auf einen lokalen Eisstausee begrenzt waren hangende Ablagerungen, wie sie besonders von HaRTMANN (1988) und LENHART (1989) detailliert aufgenommen werden konnten. Über einem, nur im Randbereich des ehemaligen Sees auftretenden, bis ca. $2 \mathrm{~m}$ mächtigen Fein- bis Mittelsand, der als Nachschütt-Schmelzwassersand gedeutet werden kann, folgten bis $15 \mathrm{~m}$ mächtige Beckensedimente. Meist direkt über unverwittertem Geschiebemergel einsetzend, war eine Grenzziehung zu diesem nur durch das Fehlen von Geschieben möglich. Die untersten Dezimeter dieser glazilimnischen Ablagerungen wiesen nur eine undeutliche Feinschichtung auf und bestanden wohl hauptsächlich aus aufgearbeitetem, dunklem, schluffig-tonigem Grundmoränenmaterial. Nach oben ging dieses kalkhaltige Sediment in einen kalkfreien Warvit über, der aus einer Wechselfolge von Lagen aus dunkelgrauem, stark tonigem Schluff $(0,2-1,0 \mathrm{~cm}$ Mächtigkeit) und etwas hellerem, feinsandigem Schluff $(0,1-1,0$ mm Mächtigkeit) bestand. Noch weiter nach oben hin deutete eine graduelle Aufhellung der dunklen Farbe zu braun bis hellbraun, verbunden mit einer schwachen Vergröberung des Materials, auf eine abnehmende Beteiligung pelitreichen Grundmoränenmaterials hin. Gleichzeitig erhöhte sich die Mächtigkeit der feinsandigen Lagen auf 1 bis $2 \mathrm{~cm}$. Vereinzelte "drop-stones“ in den basalen Metern wiesen auf driftende Eisschollen hin; von HARTMANN (1988) wurden Arthropodenspuren beobachtet. TscheE (1991) erwähnte lang aushaltende, weiße bis beigefarbene Mittelsandlagen von 10-20 $\mathrm{cm}$ Mächtigkeit, die als Schmelzwassersandhorizonte gedeutet werden können. Aus dem mittleren Teil der Warvitserie liegen aus verschiedenen Abbauschnitten unterschiedliche Beobachtungen vor. ELSNER (1987) konnte einen bis $5 \mathrm{~m}$ mächtigen, dunkelbraunen Beckenschluffkomplex beproben. Untersuchungen an der Fachhochschule Nordostniedersachsen zeigten, daß die Färbung jedoch nicht auf einen erhöhten Humusgehalt mit etwaiger Pollenführung, sondern auf fein verteilten Kohlenstaub zurückzuführen war. HARTMANN (1988) fand eine bis $5 \mathrm{~m}$ breite und $1 \mathrm{~m}$ tiefe Rinne aus grobklastischem, wohl glazifluviatil abgelagertem Material. Im Beobachtungszeitraum von LENHART (1989) fielen einerseits Trockenrisse, andererseits eine an Gesteinen aus dem Elm reiche Fließerde auf.

Der überlagernde höhere Teil war im untersten Meter der unteren Warvitabfolge noch sehr ähnlich. Zum Hangenden fand eine allmähliche Zunahme der feinsandigen Anteile statt und die Lamination wurde gröber. „Drop-stones“ fehlten vollständig, 
dafür traten häufig Lagen mit regelmäßig angeordneten Strömungsrippeln und teilweise feinen Schrägschichtungslamellen (Flaserschichtung) auf. Im Topbereich setzte sich die Farbänderung von bräunlich zu grauweiß durch; es entwickelte sich eine Wechselfolge von grauweißen, reinen und grauen, schwach schluffigen Feinsandlagen. Zu den hier noch deutlicher ausgeprägten o. g. sedimentologischen Merkmalen gesellten sich Aufund Abschiebungen unterschiedlicher Richtung, die durch Kompaktions- und Sackungsvorgänge erklärt werden können. Obwohl von HarTManN (1988: 72) innerhalb der Warvitserie verschiedene Laminationsrhythmen identifiziert werden konnten, unterblieb leider eine Zählung der unterschiedlichen Warven trotz guter Aufschlußbedingungen.

Über diesen glazilimnischen Bildungen war eine sich fortsetzende Kornvergröberung sichtbar. So wurden die schluffigen Lagen immer dünner und machten grauweißen, schwach mittelsandigen Feinsanden Platz. Dieses jüngere Schmelzwassersediment war gekennzeichnet durch eingeschwemmte Lignitflitter und Kohlebröckchen, zunehmende Horizontalschichtung bei zurückgehender Schrägschichtung und Strömungsrippeln, des weiteren kleine Schluffgerölle, häufige grobsandige und kiesführende Partien sowie Kryoturbationserscheinungen. Diese v. a. in den tonig-schluffigen Lagen ausgebildeten unregelmäßigen Verknetungen, tropfenförmigen Diapire sowie schlierig-gekröseartigen Verbiegungen waren teils über das gesamte Schichtpaket verbreitet (HARTMANN 1988), teils traten sie nur ca. 0,5-1,0 $\mathrm{m}$ unter Top auf und dokumentierten dadurch einen internen Kryoturbationshorizont (Lenhard 1989). Die obersten $0,5 \mathrm{~m}$ dieser Schmelzwassersedimentfolge wurden meist von kiesigem Mittelsand gebildet, der wiederum kaum Kryoturbationserscheinungen aufwies. Gerölluntersuchungen zeigten bei völliger Entkalkung höhere Einflüsse nordischen Materials (79-90\% in der Mittelkiesfraktion) sowie einen wesentlich geringeren Anteil an Geröllquarzen als in den älteren Schmelzwassersedimenten. Als maximale Mächtigkeiten dieser jüngeren Schmelzwasserablagerungen wurden von HARTMANN (1988) 3 m, von LENHART (1989) $5 \mathrm{~m}$ bzw. von Tschee (1991) sogar $9 \mathrm{~m}$ genannt.
An einer Stelle konnte von ElSNER (1987) der direkte Übergang der letztgenannten Schmelzwassersedimente in einen darüberlagernden Beckenschluff beobachtet werden. Unter Einschaltung von zuerst mm-dünnen, nach oben zunehmend $\mathrm{cm}$-dicken hellgrauen Schlufflagen, später einzelnen sehr gut gerundeten Grobkiesen und Steinen („dropstones") entwickelte sich ein im $\mathrm{cm}$-Bereich geschichteter, grau-braun-marmorierter, kalkfreier, ca. $2 \mathrm{~m}$ mächtiger Beckenschluff. Hierüber wiederum folgte an anderer Stelle ein bis über 2,7 m mächtiger, ungeschichteter, sehr schwach feinsandiger, stark kalkhaltiger, zum Teil bräunlich marmorierter und feinstdurchwurzelter Schluff mit besonders am Top zusammengeschwemmten Kalkkonkretionen. Dieser Sedimentkörper wurde von ELSNER (1987) als Schwemmlöß gedeutet.

\subsection{Elster-Spätglazial}

Über dem erwähnten Schwemmlöß folgten ohne erkennbare Schichtlücken mehrere im Gelände v. a. farblich gut differenzierbare, durchgehend kalkfreie Beckenschluffhorizonte. Sie gelangten in einem ca. $450 \mathrm{~m}$ breiten, wenige Meter tiefen Becken zur Ablagerung. Braune bis dunkelbraune, selten dunkelgraue Farben, Wurzelreste und -röhrchen, wenige Holzstückchen und vereinzelt schwacher $\mathrm{H}_{2} \mathrm{~S}$ Geruch belegten einen hohen Humusgehalt bzw. anaerobe Verhältnisse. Bläulichgraue Farben sowie Manganoxidausfällungen in Form von Flecken und Streifen standen dagegen in Zusammenhang mit einer späteren (Pseudo-)Vergleyung. Auffälligstes Sediment dieser bis ca. $5 \mathrm{~m}$ mächtigen Beckenschluffserie war ein stark bröckeliger, schwarzbrauner schluffiger Ton („Bröckelton“). Er verzahnte sich beckenwärts mit einem mächtigen tonigen Schluff, der durch umgelagerte Manganoxide blauschwarz gefärbt war (Gley bzw. Pseudogley) und sich ebenfalls teilweise durch eine starke Klüftigkeit (Brockigkeit) auszeichnete. Zerbröckelung und Bodenbildung dürften durch ein klimatisches Ereignis hervorgerufen worden sein. Hierbei könnte es sich um eine länger andauernde, wahrscheinlich sogar überregionale Dürre gehandelt haben, die am Uferbereich zu einer Austrocknung und im zentralen Beckenbereich zu einer intensiven (Pseudo-)vergleyung führte (ElSNer 1987). Zu demselben Ergebnis kam FeEsER (1986), der aus einem Horizont 
des Lauenburger Tons, einem Spätelster-zeitlichen Äquivalent der hier beschriebenen Schichtenabfolge, ein ähnliches Gefüge beschrieb.

Wenige Dezimeter über diesem Bröckelton war in einer hangenden, durchschnittlich $1,5 \mathrm{~m}$ mächtigen, insgesamt humusreichen Abfolge eine erste, bis $21 \mathrm{~cm}$ mächtige, dunkelbraune Torfmudde ausgebildet. Palynologische Untersuchungen erlauben Rückschlüsse auf relativ kühle und trockene Klimaverhältnisse während eines durch Kiefern, Birken und Fichten dominierten Interstadials (Offleben-IInterstadial). Als lokale Komponente trat später verstärkt die Erle als Anzeiger für eine zunehmende lokale Versumpfung auf. Ein ausführliches Pollendiagramm ist in UrBAN et al. (1988) zu finden.

Am Top belegte die starke Zunahme von tertiären Sporomorphen eine wachsende Verschwemmung durch eine zurückgehende Pflanzendecke. Sedimentologisch wurde diese Klimaverschlechterung durch bis $2 \mathrm{~m}$ mächtige, stark kalkhaltige, niveofluviatile Ablagerungen angezeigt, die bis in den ufernahen Beckenbereich geschüttet wurden. Ihr Geröllinhalt wies auf eine Zunahme der Erosion im Elm hin (N:M:P-Verhältnis $=58: 37: 5$ in ELSNER 1987). Ein von HarTMANN (1989) in ähnlicher Lage vorgefundenes, beprobtes und von ihm als fluviatil gedeutetes, stark kryoturbat beeinflußtes Sediment kann vermutlich aufgrund der von ihm ermittelten Geröllkennwerte (N:M:P-Verhältnis = 58:35:7) mit der erstgenannten grobklastischen Ablagerung parallelisiert werden.

Nach diesem sowohl sedimentologisch als auch paläobotanisch nachweisbaren Klimarückschlag wurden erneut limnische Schluffe sedimentiert. Hierbei handelte es sich um einen bis $3 \mathrm{~m}$ mächtigen, bräunlichen Beckenschluff mit zwei bis je 20 $\mathrm{cm}$ mächtigen Torfmuddelagen und einzelnen Torflinsen. Im höheren Teil der Abfolge war der Schluff stark kalkhaltig ausgebildet. Dieser Kalkgehalt wurde u. a. durch zahllose, meist gut erhaltene Gastropoden- und Bivalvenschalen verursacht. Zusätzlich fanden sich große Mengen kleiner Holzreste, Früchte und Samen. Palynologisch ähnelt dieses Offleben-II-Interstadial mit seiner Kiefern-, Birken- und Fichtendominanz stark dem Offleben-IInterstadial (URBAN et al. 1988), das somit v. a. sedimentologisch zweigeteilt erscheint.
Darauffolgend muß es erneut zu einer Klimaverschlechterung gekommen sein. Auf weiten Strecken des Tagebaus waren bis ca. $7 \mathrm{~m}$ mächtige, gelbliche, kalkfreie, feinkörnige Sande mit Kieslagen im oberen Teil und einer ca. $1 \mathrm{~m}$ mächtigen, braunen Schlufflage im Basisbereich aufgeschlossen. Wegen des raschen Abbaufortschritts in diesem Tagebaubereich, verbunden mit Hangrutschungen, konnten leider keine genaueren Informationen gewonnen werden.

Aus bzw. über dieser Feinsandserie entwickelte sich eine weitere, bis $4 \mathrm{~m}$ mächtige Beckenschluffserie mit Einlagerungen von Schluff- und Torfmudden sowie Torffetzen, aber auch Torflagen. Der braunschwarze bis hellgraue, $\mathrm{z}$. T. aber auch bläulichgraue Beckenschluff enthielt wechselnde Anteile von Ton und Feinsand bei fast völliger Karbonatfreiheit (ELSNER 1987). In den stärker organischen Abschnitten wurden Molluskenschalen, Holzreste und Mammalia-Knochenreste nachgewiesen (URBAN et al. 1991). Durch Pollenanalyse konnten über einem sterilen Basisabschnitt ein trockenes und kühles Interstadial (Esbeck-Interstadial) mit Kieferndominanz, Birken- und Fichtenbeteiligung, aber auch Anteilen von Wacholder, Weide, Tanne, Eiche, Hasel und vielen anderen Arten nachgewiesen werden. Ein detailliertes Pollendiagramm ist in URBAN et al. (1988 bzw. 1991) wiedergegeben. Die limnische Sedimentation endete durch Einschaltungen sandig-kiesigen Materials im Hangenden.

Über den limnischen Ablagerungen folgten bis $3 \mathrm{~m}$ mächtige Fließerden, in denen von der Basis zum Top eine deutliche Kornvergröberung und Karbonatzunahme festgestellt wurde. So war erst feinkörniger Sand umgelagert worden, der wahrscheinlich aus Schmelzwassersanden oder Schichten der „Feinsand-Serie“ stammte. Intensive Kryoturbationserscheinungen (ELSNER 1987, HARTMANN 1988) belegten diesen deutlichen Klimarückschlag. Im höheren Teil dieser solifluidalen Ablagerung nahm der grobklastische Anteil durch Zufuhr von Material aus den Elmhanglagen zu. Auch in diesem Abschnitt nachweisbare starke kryoturbate Verwürgungen belegten noch einmal periglaziäre Verhältnisse am Ende des Elster-Pleniglazials.

Im Hangenden folgende, stets durch eine Diskor- 
danz getrennte Holstein-zeitliche Ablagerungen waren durch Pollenuntersuchungen immer sicher stratigraphisch einzuordnen.

\section{Allertal im Raum Morsleben-Beendorf}

Das Deckgebirge des Endlagers für radioaktive Abfälle Morsleben (ERAM) war bereits mehrfach Gegenstand umfangreicher geowissenschaftlicher Untersuchungen. Nach einem Rammkernsondierprogramm folgte zwischen November 1994 und September 1995 eine dritte große Bohrkampagne, in der Bohrungen bis $750 \mathrm{~m}$ Teufe niedergebracht wurden. Besonders in der Allertalstruktur zwischen Lappwald im Südwesten und Weferlinger Triasplatte im Nordosten wurden in zahlreichen Bohrungen bis $96 \mathrm{~m}$ mächtige Quartärprofile erfaßt. Der durchschnittliche Kerngewinn in dem fast ausschließlich im Rammkernbohrverfahren (101 mm Durchmesser) erfaßten Quartär betrug deutlich über $90 \%$. Die geowissenschaftliche Aufnahme und Probenentnahme für Analysen der Korngröße, des Polleninhaltes, der $\mathrm{CaCO}_{3}$ - und Corg-Gehalte, der Geröllführung, der Morphometrie und des Geschiebeinventars nach TGL 28232 (Fraktion 4-10 $\mathrm{mm}$ ) erfolgte durch Geologen eines Ingenieurbüros und der Bundesanstalt für Geowissenschaften und Rohstoffe, Außenstelle Berlin.

Inhalt der folgenden Ausführungen sind die aus den Kernaufnahmen und o.g. Analysen vorliegenden Ergebnisse und Beobachtungen zum Geschehen während der Elster-Eiszeit in diesem Raum.

\subsection{Elster-Pleniglazial}

Die Vorgänge im Allertal vor Überfahrung durch das Elster-Inlandeis können nur durch Beobachtungen in wenigen Bohrungen rekonstruiert werden. Präglaziale Schotter, wie sie im Allertal weit verbreitet sind, treten ausschließlich in höheren Lagen unter meist geringer Bedeckung aus glazialem Quartär auf (BGR 1993). So finden sich als älteste Elster-glaziale Sedimente häufig Reste von Fließerden aus oberkretazischen Sanden, Keupergesteinen und einzelnen ersten, nordischen, gut gerundeten Geröllen. Diese Fließerden werden von geringmächtigen Beckenschluffen und bis $2 \mathrm{~m}$ mächtigen, feinkörnigen Vorschütt-Schmelzwassersanden überlagert. Durch ihre gute Klassierung und ihre helle Färbung belegen sie die Aufarbeitung der weit verbreitet unter dem Quartär anstehenden Quarzsande des Maastrichtium. Die geringe Geröllführung spricht für eine noch große Entfernung des Elster-Inlandeises.

Durch den zu dieser Zeit im Allertal vermutlich noch vorliegenden flachen Talgrund mit mächtiger Sandbedeckung, einzelnen Höhenrücken aus angewitterten Keupergesteinen sowie zahlreichen Subrosionssenken fand das vorrückende Eis ein sehr differenziertes Relief vor. Die weit verbreiteten bunten Mergel-, Ton- und Siltsteine des Mittleren Keuper wurden exariert und nach kurzem Transportweg, zusammen mit groben Schmelzwasserablagerungen und Geschiebemergelfetzen, selten auch -bänken in Form von markanten Lokalmoränen wieder abgelagert. Die Mächtigkeit dieser bunten, zum Teil auch olivfarbenen Lokalmoränen schwankt zwischen 3 und über $11 \mathrm{~m}$. Sie treten nur an wenigen, nicht korrelierbaren Stellen im Allertal, jedoch stets an der Basis der ältesten Elster-Grundmoräne auf. Die innerhalb der wechselnd kalkhaltigen Lokalmoränen häufig nachweisbaren Blöcke aus Lokalmaterial erreichen bis $40 \mathrm{~cm}$ Durchmesser; die Größe der nordischen Gerölle, untergeordnet auch Geschiebe, ist dagegen wesentlich geringer.

Die hangende, weitflächig über das Allertal verbreitete, normal ausgebildete Grundmoräne des ältesten Elster-Inlandeisvorstoßes ist als schwarz- bis dunkelolivgrauer, schluffig-feinsandiger Geschiebemergel mit reicher nordischer Geschiebeführung ausgebildet. Schmelzwassersandlinsen und eingeschuppte, häufig steil stehende Beckenschluffeinlagerungen geringer Mächtigkeit sind nicht selten. Zusammen mit scharf begrenzten olivfarbenen, stark tonig-schluffigen Geschiebemergelbereichen in $\mathrm{cm}$ - bis $\mathrm{m}$-Stärke ergibt sich das Bild eines eher unruhigen Eisvorstoßes. Bemerkenswert ist, daß in zahlreichen, zum Teil weit auseinanderliegenden Bohrungen im Allertal, drei durch Zwischenmittel deutlich getrennte Geschiebemergelbänke des ältesten Elster-Eisvorstoßes unterschieden werden können. Hierbei basiert die stratigraphische Zuordnung auf der durch palynologische Untersuchungen gesicherten Überdeckung durch Holsteinzeitliche Sedimente einerseits und auf der in stets gleicher Höhenlage und lithologischer Ausbildung 


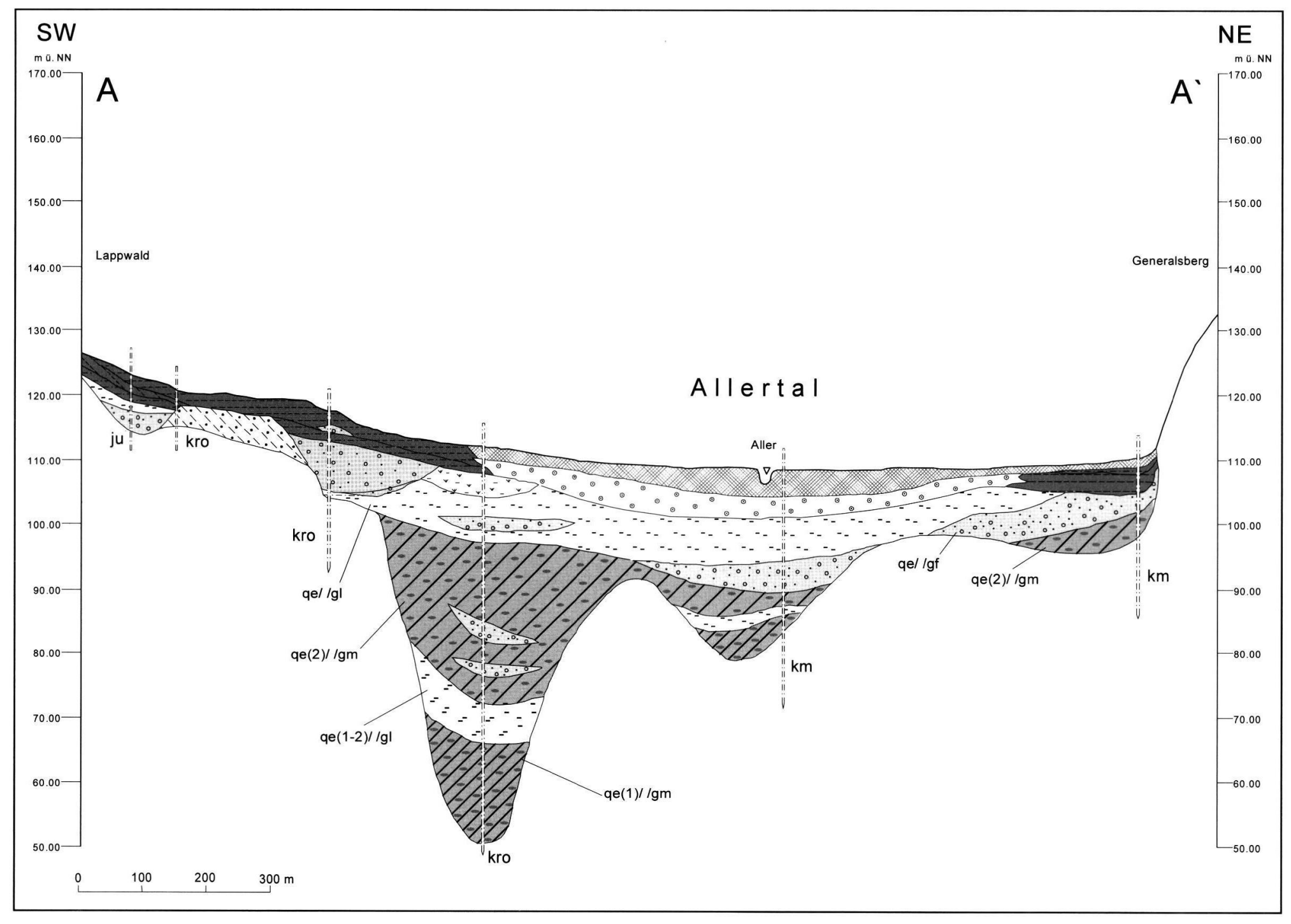


auftretenden hangenden jüngeren Elster-Grundmoräne sowie den mit ihr verbundenen Schmelzund Stillwassersedimenten andererseits. Besonders innerhalb der mit meist mächtigen Elster-zeitlichen Sedimenten ausgefüllten Subrosionssenken (s. Abb. 3), aber nicht nur dort, ist die o. g. Dreiteilung gut zu erkennen.

Nach Auswertung aller verfügbaren Daten und Beobachtungen zeigt sich folgendes Bild:

Jede der drei Geschiebemergelbänke beginnt im Idealfall an der Basis mit einem oliv- bis dunkelolivgrauen, stark schluffig-tonigen, sandarmen, sehr homogenen und kompakten Geschiebemergel, der durchschnittlich 1 bis $2 \mathrm{~m}$, maximal jedoch auch bis über $5 \mathrm{~m}$ Mächtigkeit erreichen kann. Neben der genannten Färbung, der spezifischen Lithologie und dem vollständigen Fehlen von Schmelzwassersandeinschaltungen ist auch das Auftreten von überwiegend einheimischen Komponenten (speziell Buntsandsteine und grünlich-bunte Mergelsteine des Mittleren Keuper) sehr typisch und damit eine bereits makroskopische Einordnung sehr leicht möglich. Teils mit sehr scharfer Grenze, teils aber

Abb. 3: Profilschnitt A - A' (zwischen Beendorf und Morsleben) durch das Quartär im mittleren Allertal. Zu beachten sind die tiefen Subrosionssenken, die mit Sedimenten des ersten und zweiten Elster-Eisvorstoßes in diesem Raum ausgefüllt wurden. Im Hangenden innerhalb des Talraumes gleichmäßige Verbreitung jüngerer Bildungen (Holstein bis Holozän).

Fig. 3: Cross-section $A-A^{\prime}$ (between Beendorf and Morsleben) showing the deposits of Quaternary age in the middle Aller valley. Notice deep depressions caused by salt leaching filled with sediments of the first and second Elsterian ice advance in this area. In the valley floor above uniform distribution of younger deposits (Holsteinian to Holocene).

Legende:

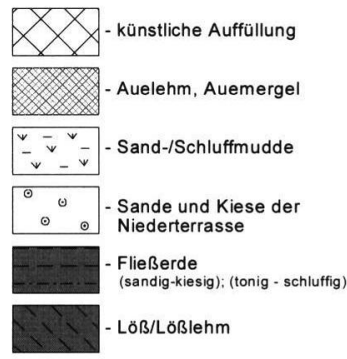

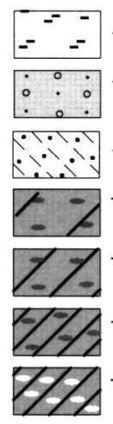

- Beckenschluff ( // gl - I) - glazifluviatile Sande und Kiese ( // gf)

- Geschiebemergel/-lehm Drenthe-Stadial

- Grundmoräne (qe(3?)) jüngstes Elster-Stadial

- Grundmoräne (qe(2)) jüngeres Elster-Stadial

- Grundmoräne (qe(1)) ălteres Elster-Stadial

- Lokalmoräne (qe(1)) alteres Elster-Stadial auch durch bis maximal $30 \mathrm{~cm}$ mächtige, wohl inglaziär sedimentierte Mittel aus glaukonitischen Feinsanden, umgelagerten Schluffen oder weißgrauer Grundmoräne getrennt, folgt ein völlig anders ausgebildeter hangender Bereich. Hierbei handelt es sich um einen typisch feinsandig-schluffigen, schwarz- bis dunkelolivgrauen, ungeschichteten Geschiebemergel mit einzelnen Schlieren, Linsen und selten Lagen von Schmelzwassersedimenten. Besonders in den hangenden Abschnitten nimmt die Mächtigkeit und Häufigkeit dieser Einschaltungen jedoch deutlich zu; sie sind zudem teilweise chaotisch gelagert. Der Geschiebebestand ist durchgehend eindeutig nordisch bestimmt, vereinzelt treten Ligniteinschlüsse auf. Die Mächtigkeit dieser hangenden Abschnitte variiert sehr stark und beträgt zwischen 2 und $9 \mathrm{~m}$.

Aufgrund der genannten lithologisch-sedimentologischen Merkmale wird der basale olivfarbene Abschnitt der einzelnen Geschiebemergelbänke als typischer „lodgement till“ gedeutet, der durch gletscherbasisnahe Durchmischung von lokal anstehendem Material (Keuper, Buntsandstein) entstand. Er stellt damit eine Übergangsform (Lokalfazies) zwischen der bereits beschriebenen Lokalmoräne und einer „normalen“ Grundmoränenfazies, v. a. mit aus größerer Entfernung bis aus Skandinavien heran transportiertem Material dar. Dieser Normalfazies entspricht der obere schwarzolivgraue $\mathrm{Ab}$ schnitt der einzelnen Geschiebemergelbänke. Möglicherweise handelt es sich hierbei basal ebenfalls um einen „lodgement till“, der aber nach oben bald in einen „melt-out till“ übergeht, wie er beim Abschmelzen von Inlandeismassen sedimentiert wird.

Zwischen den einzelnen Geschiebemergelbänken treten sowohl fein- bis grobkörnige, meist kiesarme Schmelzwassersande, aber auch nicht weniger häufig deutlich geschichtete Beckenschluffe von Dezimetern bis 6 Metern Mächtigkeit auf. Vereinzelt sind diese Zwischenmittel glazitektonisch überprägt, so daß zum Teil eine gesicherte Beweisführung der ursprünglichen Lagerungsverhältnisse erschwert ist. In anderen Kernen ist jedoch über viele Meter vertikaler Erstreckung das Studium zahlreicher ungestörter sedimentologischer Feinstrukturen, wie Rippelmarken, Belastungsstrukturen oder Eindruckmarken von "drop-stones“ in horizontal geschichteten Beckenschluffen und 
-feinsanden möglich. Die Wahrscheinlichkeit, daß es sich bei allen beobachteten Zwischenmitteln stets um vom Gletschereis aufgenommene und bald wieder sedimentierte Schollen handelt, ist daher sehr gering. Vielmehr kann davon ausgegangen werden, daß das Untersuchungsgebiet während des ersten Hauptvorstoßes des Elster-Inlandeises mehrmals vom Gletscher überfahren wurde. Dies bedeutet, daß es in diesem Außenbereich des Eisschildes während eines ältesten Hauptvorstoßes zu mindestens drei Oszillationen kam. Geht man weiterhin davon aus, daß zumindest einige wenige der in den einzelnen Zwischenmitteln auftretenden sehr geringmächtigen Grundmoräneneinschaltungen auf kurzfristige Vorstöße lokaler Gletscherzungen und nicht nur auf Umarbeitung liegenden Grundmoränenmaterials zurückzuführen sind, ergibt sich eine Reichweite der Oszillationsbewegungen maximal im $10 \mathrm{er} \mathrm{km}$-Bereich.

Im Gegensatz zu der durch lokale Subrosionssenken geprägten Verbreitung der Grundmoränen des ältesten Elster-Eisvorstoßes findet sich das hangende Zwischenmittel im Liegenden der jüngeren ElsterGrundmoränen, von einer Subrosionssenke abgesehen, im Allertal in einem relativ durchgehenden Höhenniveau von ca. 90-100 m ü. NN (s. Abb. 3). Bei diesem Zwischenmittel handelt es sich um wenige Dezimeter bis über $11 \mathrm{~m}$ mächtige Schmelzwassersande und -kiese, die nach oben hin in der Regel feinkörniger werden. In diesen hellbraungrauen bis hellolivfarbenen, kalkhaltigen Fein- bis Mittelsanden, selten Beckenschluffen, sind kohlige Flitter weit verbreitet. Eine ruhige Horizontalschichtung im $\mathrm{mm}$ - bis $\mathrm{cm}$-Bereich spricht für eine größere Entfernung des Inlandeises. In einer Bohrung (Dp Mors 33B) ist in stark kalkhaltigen, feinsandig-tonigen Beckenschluffen unter fraglichem Geschiebedecksand eine geringmächtige Bodenbildung angedeutet.

Nur in wenigen Bohrungen wurden hangende, bis maximal $4 \mathrm{~m}$ mächtige, wieder etwas gröberkörnige Schmelzwassersande angetroffen, die als VorschüttSchmelzwassersedimente des erneut vorrückenden Gletschers gedeutet werden müssen. Die in ihnen auftretenden Kiese sind stark nordisch geprägt (N:M:P-Verhältnis bzw. F/NK-Quotient der Mittelkiesfraktion $=91: 7: 2$ bzw. 0,43). Bei einer Durchmusterung wurde unter den wenigen Grob- kiesen ein Faxekalkstein gefunden, der Rückschlüsse auf einen westskandinavischen Einfluß der Geschiebeführung des Gletschers zuläßt. Der einheimisch-mesozoische Anteil ist v. a. durch Gesteine des Buntsandstein und des Unterrhät vertreten. In einigen Bohrkernen treten am Top des Hauptzwischenmittels Verwürgungen und kleine Eiskeilpseudomorphosen auf.

Der hangende Geschiebemergel besitzt an seiner Basis häufig eine bis wenige Meter mächtige Zone mit $\mathrm{mm}$ - bis cm-dünnen Lagen aus vom Eis abgescherten und dann ausgewalzten Schmelzwassersanden oder Beckenschluffen. In einem Fall sprechen senkrecht stehende Geschiebe in einer stark tonigen Matrix für eine lokale subaquatische Ablagerung der tiefsten Teile der Grundmoräne. In einer anderen Bohrung konnten farblich deutlich unterscheidbare, olivfarbene Schlieren von älterem Geschiebemergel (s. o.) nachgewiesen werden. Sieht man von diesen, auf die Basisbereiche begrenzten Erscheinungen $a b$, ist der jüngere Geschiebemergel im Gegensatz zu den älteren durchgehend homogen und kompakt ausgebildet. Er besitzt durchgehend einen hohen Kalkgehalt, eine feinsandig-schluffige Matrix, eine dunkel- bis schwarzolivgraue Farbe, eine durch nordische Komponenten geprägte Geschiebeführung sowie eine Mächtigkeit von wenigen bis in Subrosionssenken durchschnittlich 20-30 m. Im Vergleich zu den älteren Geschiebemergeln ist der Xylitgehalt erhöht. Olivgrüne, tonige, durch einheimische Gesteine geprägte Bereiche treten nicht auf; intramoränale Schmelzwassersandlinsen wurden sehr selten durchbohrt. Aufgrund dieser Merkmale scheint es sich um den „lodgement till“ eines weit nach Süden vorgestoßenen Inlandeises zu handeln, das das Untersuchungsgebiet im Zeitraum seiner Maximalausdehnung mit großer Mächtigkeit überdeckte.

Weit verbreitet sind ganz am Top dieses ansonsten homogenen, ungeschichteten Geschiebemergels sehr unterschiedlich mächtige Lagen, Schlieren und Linsen von Sand. Diese sind verbunden mit einer nach oben zunehmenden chaotischen Lagerung typische Merkmale von Ablationsmoränen, wie sie vereinzelt auch in den Dachbereichen der liegenden Grundmoränenbänke angetroffen wurden. Die maximal nachgewiesene Mächtigkeit einer solchen Ausschmelzmoräne wurde in der Bohrung Dp 
Mors 69A mit 4,4 m bestimmt, wo sie eine $29 \mathrm{~m}$ mächtige, ungeschichtete Absetzmoräne überlagerte.

Während fast überall im zentralen Allertal im Hangenden dieser jüngeren Elster-Grundmoräne Nachschütt-Schmelzwassersedimente oder auch mächtige Beckenbildungen angetroffen werden können (s. Abschnitt 5.2), bieten die Kerne der Bohrung Dp Mors 86A ein völlig anderes Bild. Der Ansatzpunkt dieser Bohrung lag auf einem östlich der Allerniederung gelegenen Plateau bei 136,80 m ü. NN und damit ca. $25 \mathrm{~m}$ über den anderen Bohransatzpunkten im zentralen Allertal (s. Abb. 4). Durch einen in diesem Bereich nach Westen mäandrierenden Allerflußbogen, blieb dieses Plateau von stärkerer Erosion verschont und liefert damit heute, gleich einem Zeugenberg, Hinweise auf die ursprüngliche, andernorts bereits abgetragene pleistozäne Schichtenfolge im Untersuchungsraum.

In der gleichen absoluten Höhenlage um $100 \mathrm{~m}$ ü. NN wie in allen anderen Bohrungen, wurde auch in der Bohrung Dp Mors 86A der oben beschriebene jüngere, hier allerdings nur $1,5 \mathrm{~m}$ mächtige Elster-Geschiebemergel angetroffen. Hierüber folgen an dieser Stelle $4 \mathrm{~m}$ mächtige, stark kalkhaltige, wechselnd grobsandig-kiesige, ungeschichtete Schmelzwasserablagerungen mit kleinen Nestern und Schlieren von Geschiebemergel an ihrem Top. Im unteren Drittel tritt dagegen als Einschaltung ein inhomogenes, ungeschichtetes Gemenge aus zersetzten, in benachbarten Hanglagen anstehenden Fest- und Lockergesteinen mit vereinzelten nordischen Kiesen auf, das als Fließerde gedeutet werden kann.

Bedeutsamer ist jedoch das über den Schmelzwasserablagerungen durchbohrte Sediment, bei dem es sich zweifelsfrei um eine weitere und damit jüngste Elster-Grundmoräne handelt. Diese 8,9 m mächtige ungeschichtete Grundmoräne besitzt eine feinsandig-schluffige Matrix, einen durchgehend hohen Kalkgehalt und eine schwarz- bis dunkelolivgraue Farbe. Makroskopisch sind keinerlei Unterschiede zu den liegenden, ähnlich farbigen, älteren Grundmoränenbänken erkennbar. Auch an der Basis dieses jüngsten Elster-Geschiebemergels treten nach unten zunehmend Lagen, Schlieren und Nester von braungrauen, schwach kalkhaltigen
Schmelzwassersanden auf. In den obersten Dezimetern nachweisbare, stark verbrodelte Nester und Schlieren von Schmelzwassersanden sprechen für eine intensive kryoturbate Beeinflussung des als Ablationsmoräne abgelagerten obersten Abschnitts dieser Grundmoränenbank. Leider wurden aus dieser jüngsten Elster-Grundmoräne weder Proben für Korngrößenanalysen noch für Grobgeschiebezählungen nach der TGZ-Methode entnommen.

Die im Hangenden dieses jüngsten ElsterGeschiebemergels in der Bohrung Dp Mors 86A folgende, über $8 \mathrm{~m}$ mächtige limnische bis glazilimnische Beckenfolge (unterhalb ebenfalls außergewöhnlich mächtiger Vorschütt-Schmelzwassersedimente und Grundmoräne des Drenthe-Stadiums der Saale-Eiszeit) ist mit weit verbreiteten ähnlichen Sedimenten im Hangenden des zweiten Elster-Geschiebemergels im zentralen Allertal gut vergleichbar (s. Abschnitt 5.2). Da diese vergleichbaren Sedimente dort jedoch durchschnittlich $15 \mathrm{~m}$ tiefer anstehen, kann hierdurch auf einen enormen Schmelzwasseranfall am Ende des Elster-Glazials geschlossen werden. Verbunden mit einer sich ausbildenden, tief einschneidenden Vorform der Aller führte dies zu einer starken Erosion der ursprünglich weit mächtigeren Elster-zeitlichen Schichtenfolge.

Wenige Dezimeter mächtige, horizontal lagernde, fein- bis mittelkörnige, schwach kiesführende, nach oben abnehmend kalkhaltige Schmelzwassersande sind im Allertal unregelmäßig verbreitet und können als Erosionsreste einst mächtigerer NachschüttSchmelzwassersedimente gedeutet werden. Ebenso wie in den nur in einer Bohrung darüber lagernden 1,3 m mächtigen, hellbraungrauen bis olivgrauen, kalkhaltigen, feinsandig-schluffigen Beckenbildungen treten in ihnen vereinzelt kryoturbate Verwürgungen auf. Es sind dies die jüngsten Bildungen, die sicher in das Elster-Pleniglazial gestellt werden können.

\subsection{Elster-Spätglazial}

Die Verhältnisse im Elster-Spätglazial waren, wie bereits angeführt, im Allertal durch einen hohen Anfall von Schmelzwasser, verbunden mit den 


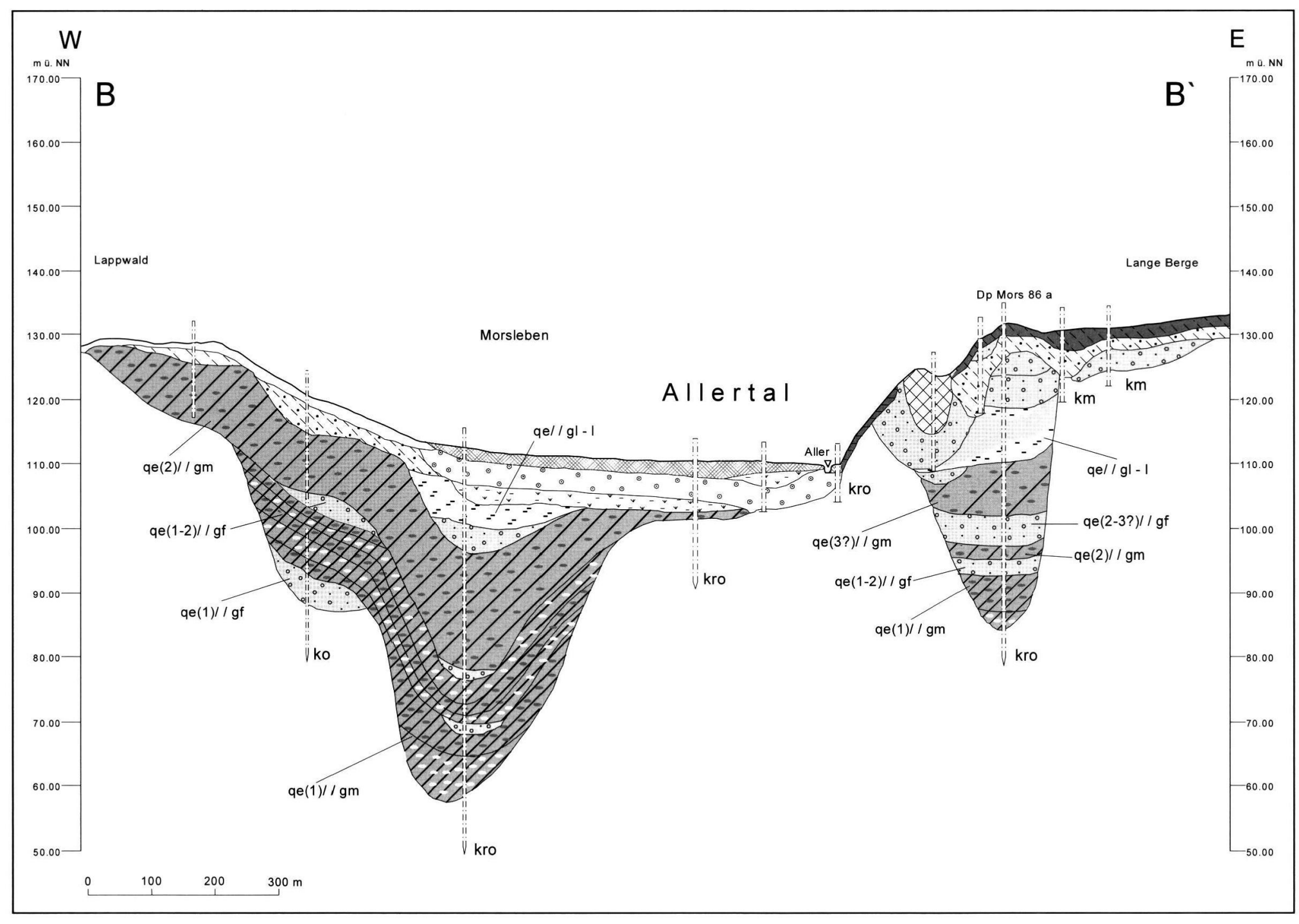


Wirkungen einer sich schnell ausbildenden und einschneidenden frühen Aller geprägt. In zahlreichen Toteislöchern kamen teils bis über $10 \mathrm{~m}$ mächtige Beckenschluffe und -feinsande zur Ablagerung. An ihrem Top leiten diese zum Teil mit anscheinend fließendem Übergang in ebenfalls mehrere Meter mächtige Holstein-zeitliche limnische Schluffe, Mudden, Torfe sowie fluviatile Sande über, die im gesamten zentralen Allertal weit verbreitet sind.

Obwohl die Spätelster-zeitlichen Ablagerungen im Detail sehr unterschiedlich ausgebildet sind, lassen sich doch, allerdings mit gewissen Unsicherheiten, großräumige Korrelationen vornehmen.

Abb. 4: Profilschnitt B - B' (bei Morsleben) durch das Quartär im südlichen Allertal. Im westlichen Talraum (ähnlich wie im Profilschnitt $A-A^{\prime}$ ) eine tiefe Subrosionssenke, die mit Ablagerungen des ersten und zweiten Elster-Eisvorstoßes ausgefüllt wurde. Im östlichen Talraum sind dagegen an der Talschulter zusätzlich Sedimente eines dritten Elster-Eisvorstoßes und Spätelsterzeitliche Bildungen erhalten geblieben. Ihr Vorhandensein und ihre Höhenlage belegen neben einer dritten Elster-Inlandeisüberfahrung dieses Raumes, dass im zentralen Aller-Talraum im Zeitraum zwischen Abschmelzen des Elster-Inlandeises und Erreichen des Klimaoptimums der Holstein-Warmzeit mindestens $15 \mathrm{~m}$ Sediment abgetragen wurden. Alle jüngeren fluviatilen und limnischen Sedimente des zentralen Aller-Talraums lagern in dieser spät-Elster-zeitlichen Erosionsform, sind flächenhaft verbreitet und erreichen jeweils nur wenige Meter Mächtigkeit. (Legende vgl. Abb. 3)

Fig. 4: Cross-section B - B' (near Morsleben) showing the deposits of Quaternary age in the southern Aller valley. In the western part of the valley there is a deep depression caused by salt leaching (similar to cross-section A- A') filled with deposits of the first and second Elsterian ice advance. Additionally at the eastern valley shoulder sediments of a third Elsterian ice advance and deposits of late Elsterian age are observable. Their existence and their altitude are proof of a former third Elsterian ice advance in this area as well as an erosion of more than 15 $\mathrm{m}$ of sediments between thawing of the Elsterian ice sheet and the climatic optimum of the Holsteinian. All younger fluvial and limnic sediments of the central valley floor rest in this erosion form of late Elsterian age. Distribution of these younger sediments is uniform with thicknesses of only several meters each. (legend like fig. 3)
Meist direkt aus den genannten NachschüttSchmelzwasserbildungen entwickeln sich erst mittel-, dann immer feinkörnigere, nach oben lagenweise schwach schluffige und glimmerführende, kalkhaltige, gut klassierte Sande. Diese söhlig lagernden, deutlich geschichteten Sande erreichen bis $3 \mathrm{~m}$ Mächtigkeit. Sie wurden teils in lokalen Becken, teils in Nebenarmen der frühen Aller sedimentiert. Ein wenige Dezimeter mächtiger, kalkfreier bis schwach kalkhaltiger Bereich mit schwachem $\mathrm{H}_{2} \mathrm{~S}$-Geruch und sehr geringen Mengen stark zersetzten organischen Materials spricht für die Ausbildung einer geschlosseneren Pflanzendecke, vielleicht in einem kühlen Interstadial. In den darüber lagernden Sanden beobachtbare schlierige Verwürgungen lassen jedoch erneut kaltzeitliche Temperaturbedingungen vermuten.

Sichere interstadiale Klimaverhältnisse sind durch bis insgesamt 3,5 m mächtige hangende Bildungen nachgewiesen, die in zahlreichen Bohrungen angetroffen wurden: Aus den basalen, größtenteils sterilen und kalkhaltigen Feinsanden entwickeln sich mit fließendem Übergang bis $2,7 \mathrm{~m}$ mächtige, durchgehend kalkfreie, undeutlich geschichtete, hellolivgraue, fein- bis mittelkörnige Sande mit lagenweise oder diffus verteiltem organischen Material. Vereinzelt finden sich in diesen gut klassierten fluviatilen Sanden auch Fetzen umgelagerter Schluffmudde. Auf Schwellen, wo diese Sande fehlen, kann ein geringmächtiger Boden entwickelt sein.

Nach oben leiten die Sande in bis über $2 \mathrm{~m}$ mächtige limnische Bildungen über. Es handelt sich um dunkel- bis schwarzgraue, meist kalkfreie, massige bis fein geschichtete, wechselnd tonige Schluffe, zum Teil auch Schluffmudden, mit diffus verteiltem oder auch lagenweise angereichertem organischen Material (Holzstückchen, Pflanzenhäcksel) und schwachem $\mathrm{H}_{2} \mathrm{~S}$-Geruch. Bereichsweise treten massenhaft Molluskenschalenbruchstücke, aber auch zweiklappige Exemplare von kleinen Pisidien auf. Am Top dieser limnischen Bildungen findet sich in der Bohrung Dp Mors 86A (s. o.), durch einen $1 \mathrm{~m}$ mächtigen, massigen, braungrauen, schwach kalkhaltigen Beckenschluff getrennt, eine $50 \mathrm{~cm}$ mächtige Bodenbildung.

Weit verbreitet im Allertal sind auf die limnischen Schluffe folgende, bis fast $8 \mathrm{~m}$, durchschnittlich je- 
doch 3 bis $5 \mathrm{~m}$ mächtige kaltzeitliche Beckenablagerungen. Diese sehr typischen Ablagerungen beginnen mit schwach feinsandigen, lagenweise sehr schwach tonigen Schluffen und nachfolgend schwach schluffigen Tonen. Darüber lagern wieder wechselnd feinsandige Schluffe und zuletzt lagenweise schluffige oder mittelsandige Feinsande. Gemeinsam ist all diesen Sedimenten der hohe Kalkgehalt, die Feinglimmerführung, die intensive, teilweise warvitische, horizontale Schichtung im $\mathrm{mm}$ - bis cm-Bereich und das lagenweise Auftreten von sehr feinen Kohleflittern. Die wechselnd hellbis schwarzolivgraue Färbung wird bereichsweise durch starke Manganoxidausfällungen in Form von Lagen, Schlieren und Flecken überprägt. Sehr selten wurde ein sehr schwacher $\mathrm{H}_{2} \mathrm{~S}$-Geruch oder stark zersetzte kleine Pflanzenhäcksel registriert. Im obersten, gröberkörnigen Bereich finden sich vereinzelt schwache Kryoturbationen oder sehr kleine Eiskeilpseudomorphosen.

Ein Wechsel zu erneuten interstadialen Klimabedingungen ist durch unterschiedlich mächtige und verschieden ausgebildete hangende Sedimente belegt. Teils wurden erneut fluviatile, schwach kalkhaltige, dunkelgraue bis braungraue Feinsande mit lagenweise angereichertem organischen Material, teils ebenfalls schwach kalkhaltige und tonige, dunkelolivgraue, limnische Schluffe mit einzelnen Molluskenschalenbruchstücken abgelagert. In einer Bohrung (Dp Mors 68A) wurde eine $1,1 \mathrm{~m}$ mächtige, oliv- bis grüngraue, bereichsweise feingeschichtete Kalkmudde durchteuft. In ihr sind unregelmäßig verteilte Pflanzen- und senkrecht angeordnete Wurzelreste erhalten. In der Bohrung Dp Mors 86A (s. o.) ist diese Klimaverbesserung wahrscheinlich durch eine $60 \mathrm{~cm}$ mächtige Bodenbildung repräsentiert.

Durch die unterschiedlich große Diskordanz im Liegenden der Holstein-zeitlichen Sedimente ist eine Rekonstruktion des weiteren Geschehens am Ende des Elster-Pleniglazials nur lückenhaft und mit größeren Unsicherheiten behaftet möglich. Während in der Bohrung Dp Mors 68A knapp 3 $\mathrm{dm}$ mächtige niveofluviatile, stark kalkhaltige, grobklastische Sedimente den Abschluß der Elsterzeitlichen Sedimentfolge bilden, erlaubt die Bohrung Dp Mors 86A (s. o.) noch weitere Rück- schlüsse. Hier finden sich statt der niveofluviatilen Sedimente wenige Dezimeter mächtige, grobkörnige fluviatile Ablagerungen, die nach oben hin, mit gradueller Korngrößenabnahme, schnell schwach tonigen, feinsandigen, massigen und ungeschichteten, unten weißolivgrauen, oben weißbraungrauen Beckenschluffen Platz machen. Die vollständige Kalkfreiheit ist vermutlich auf eine tiefreichende Holstein-zeitliche Entkalkung zurückzuführen. In den obersten $50 \mathrm{~cm}$ dieser insgesamt 2 $m$ mächtigen Abfolge ist erneut ein deutlicher Bodenhorizont ausgebildet.

Über dieser Bodenbildung sind in der Bohrung Dp Mors $86 \mathrm{~A}$ noch $3 \mathrm{dm}$ mächtige Reste eines weißbraungrauen, ungeschichteten, schluffigen Feinsandes erhalten, der mit scharfer Grenze von mächtigen, grobkörnigen und kalkhaltigen Drenthe-IVorschütt-Schmelzwassersanden gekappt wird.

\section{Zusammenfassung und Korrelations- möglichkeiten}

\subsection{Zusammenfassende Gliederung der Elster-Eiszeit im Untersuchungsraum}

Obwohl die einzelnen Schichtglieder der Elsterzeitlichen Abfolgen im Tagebau Schöningen und in dem $13 \mathrm{~km}$ nordöstlich sich davon erstreckenden Allertal zum Teil sehr unterschiedlich ausgebildet sind, ist eine übergreifende Korrelation dennoch möglich.

Das Allertal wurde während der Elster-Eiszeit dreimal vom Inlandeis überfahren. Hierbei ist der jüngste Eisvorstoß mit großer Wahrscheinlichkeit auf eine regionale Oszillation zurückzuführen. Zusätzlich lag das Allertal auch während des ersten Eisvorstoßes im Oszillationsbereich des Gletschers. Der im Zuge des zweiten Eisvorstoßes abgelagerte homogene Geschiebemergel ist schluffig-feinsandig ausgebildet, von schwarzolivgrauer Farbe und erreicht Mächtigkeiten bis $33 \mathrm{~m}$. Er kann damit leicht mit dem zweiten Geschiebemergel aus dem Tagebau Schöningen verglichen werden, der bei gleicher sedimentologischer Ausbildung bis über 28 m Mächtigkeit erreicht. Die in diesem mächtigen Geschiebemergel durchgeführten Einregelungsmessungen belegen eine Drehung der Eisstromrichtung von NW-SE über N-S und NE-SW bis hin zu E-W. 
Ein ähnliches Verhalten wurde von RICHTER (1961) in einem Elster-Geschiebemergel in den RhätSandsteinbrüchen bei Velpke beobachtet. Auch die aus dem Schöningen-Hauptgeschiebemergel und dem Velpke-Geschiebemergel (Richter 1962, MERKT 1996) ermittelten TGZ-Werte (vgl. Abb. 6) stimmen gut überein.

Die während der ersten Eisvorstöße im Allertal abgelagerten Grundmoränenbänke besitzen häufig einen charakteristischen schluffig-tonigen, kompakten, olivgrünen Basisbereich. Auch die von Tschee (1991) im Liegenden des mächtigen Geschiebemergels im Tagebau Schöningen dokumentierte Grundmoräne ist derartig ausgebildet und kann daher vermutlich mit den ersten Eisvorstößen in diesem Raum parallelisiert werden. Gleiches trifft für die von WAGENBRETH (1970) erwähnte, maximal $5 \mathrm{~m}$ mächtige, graue bis grüngraue, tonige Varietät einer Elster-Grundmoräne in den Oscherslebener Mulden zu.

Zum Teil typisch für das Untersuchungsgebiet scheinen auch mächtige Lokalmoränen an der Basis der ältesten Elster-Grundmoränenbank zu sein, wie sie von Wiegers (1932), WaGenbreth (1970) und nun auch aus dem Allertal beschrieben wurden.

Die bisher nur in der Bohrung Dp Mors 86A im Allertal nachgewiesene jüngste Elster-Grundmoräne kann nur mit Schwierigkeiten auf lokale Rutschungsvorgänge, wesentlich wahrscheinlicher jedoch auf eine letzte, auf den Untersuchungsraum beschränkte Oszillation während der Eisabbauphase des 2. Elster-Inlandeisvorstoßes zurückgeführt werden. Ob es sich auch bei der von WaGENBRETH (1970) aus dem Basisbereich der Bode-Hauptterrasse erwähnten, bis $24 \mathrm{~m}$ mächtigen, braungrauen Grundmoräne um ein Äquivalent dieser jüngsten Grundmoräne handelt, muß weiteren Untersuchungen (s. Kap. 7) vorbehalten bleiben.

Besondere Beachtung verdienen die mächtigen Vorschütt-Schmelzwassersedimente im südlichen Untersuchungsgebiet, die v. a. mit dem zweiten Elster-Eisvorstoß in diesem Raum zu korrelieren sind. Vermutlich sind sie für die weitflächige Erosion der ältesten Grundmoränen südlich des Allertals verantwortlich. Von Norden nach Süden nimmt die Mächtigkeit dieser kiesigen Schmelzwassersande zu: im Allertal werden über $11 \mathrm{~m}$, im Tagebau Schöningen weit über $20 \mathrm{~m}$ und direkt nördlich des Großen Bruchs nördlich Hadmersleben (WIEGERS 1932) über $30 \mathrm{~m}$ Mächtigkeit erreicht. Hier und im Raum Neuwegersleben werden sie seit Jahren in großen Kiesgruben abgebaut. Aufgrund der Untersuchungen von HaRTMANN (1988) im Tagebau Schöningen handelt es sich wohl um Ablagerungen eines Vorschüttsanders. Als Vorfluter dieses Sanders und damit als eine Art Urstromtal kann das Große Bruch angenommen werden, über das eine Entwässerung nach Westen Richtung Oker erfolgt sein muß. Vom Harz zuströmende Flüsse mögen die abgeführten Wassermengen zusätzlich erhöht haben. Die kürzlich in Feldmann, Groetzner \& Weymann (2001) anders postulierte Entstehungsgeschichte des Großen Bruchs braucht hier in diesem Zusammenhang nicht diskutiert werden, da sie auf qualitativ völlig unzureichenden Bohrgutausträgen beruht (frdl. mdl. Mitt. Herr WeYMANN).

Auch die Spätelster-zeitlichen Ablagerungen aus dem Tagebau Schöningen und dem Allertal können miteinander parallelisiert werden. Hierbei ist jedoch von Bedeutung, daß im Allertal vorwiegend fluviatile Sedimente zum Absatz kamen, während die Helmstedter Westmulde bei Schöningen durch ein limnisches Ablagerungsmilieu geprägt war. So wurden im Tagebau Schöningen Ablagerungen von zwei Interstadialen überliefert, die dank guter Pollenerhaltung palynologisch sicher einstufbar waren. Hierdurch war zum ersten Mal in Mitteleuropa der Nachweis von deutlichen Klimaschwankungen am Ende des Elster-Glazials möglich (URBAN et al. 1988). Zusätzlich erscheint bei Schöningen das älteste, das Offleben-Interstadial, v. a. sedimentologisch zweigeteilt. Dieses Offleben- und das hangende Esbeck-Interstadial sind dort durch mächtige niveofluviatile Feinsande voneinander getrennt.

Auch im Allertal sind die Sedimente von mindestens zwei Spätelster-Interstadialen überliefert. Im Gegensatz zu den Beckenschluffen im Tagebau Schöningen entwickeln sich im Allertal die ältesten interstadialen Ablagerungen aus fluviatilen Sanden. In ihnen ist bereichsweise jedoch bereits organisches Material enthalten; dagegen fehlen Merkmale einer 


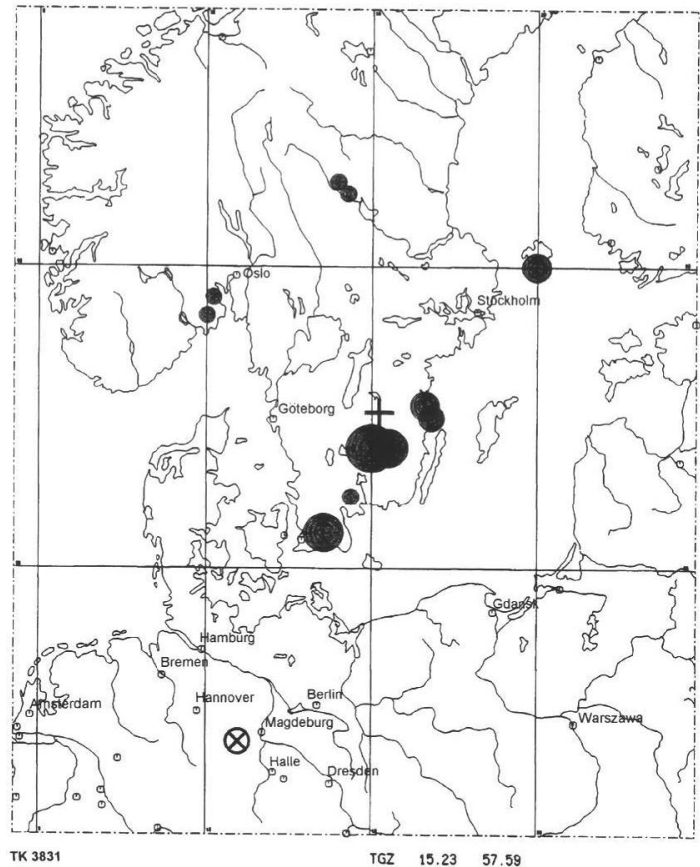

(5a)

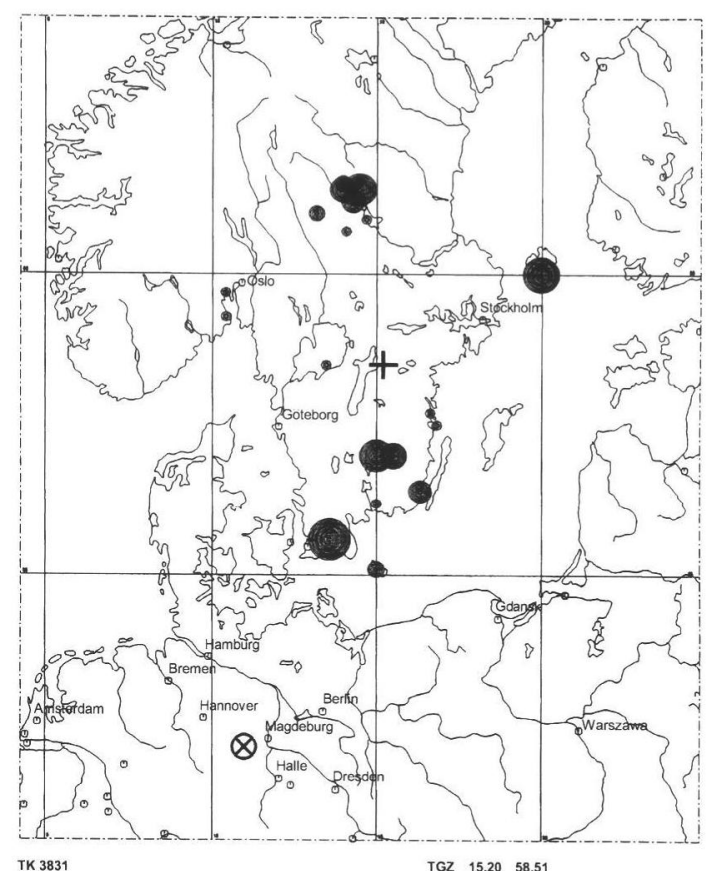

(5b)
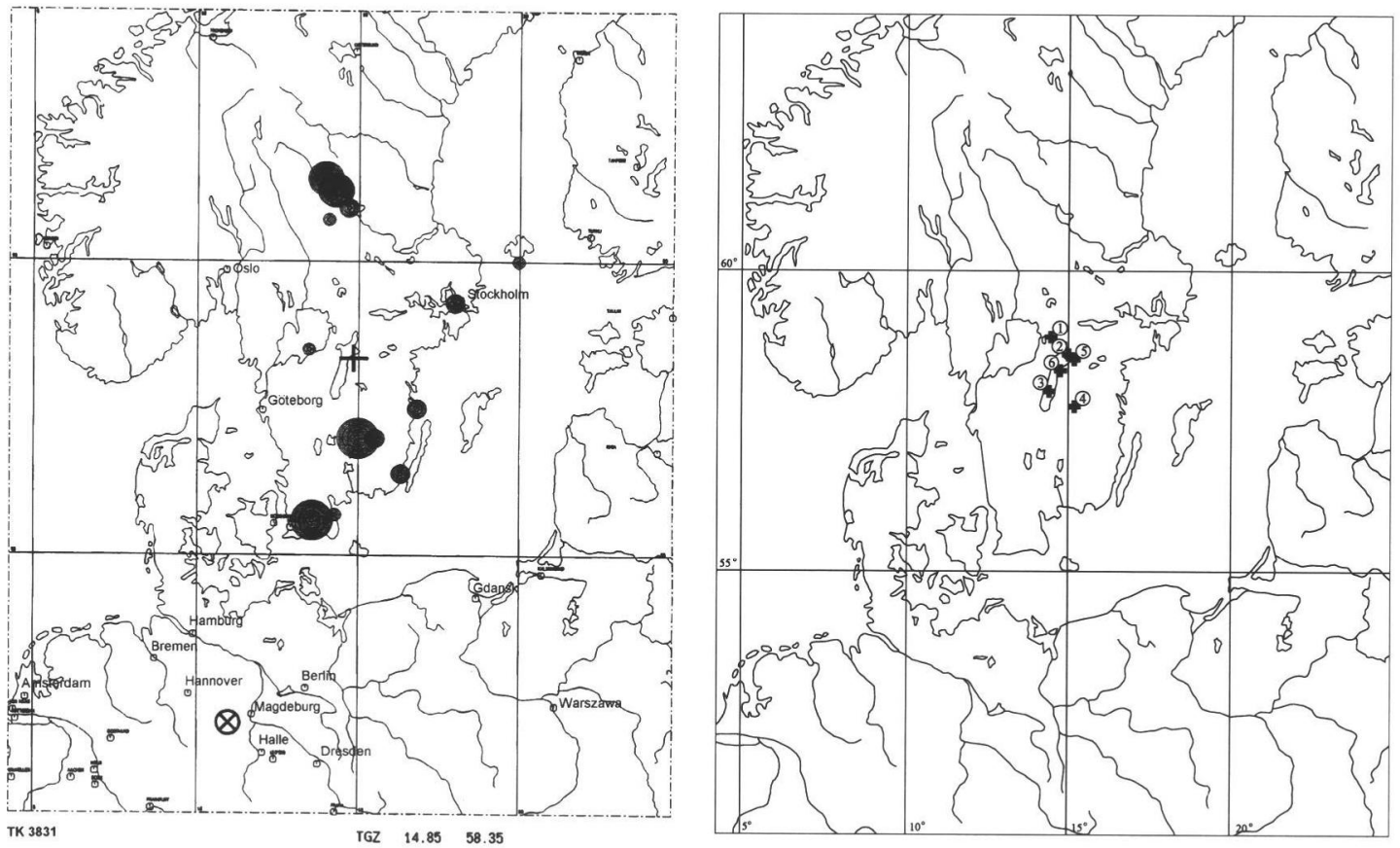

(5c) 
Trockenperiode (s. Abschnitt 4.2). Die hangenden humosen fluviatilen Sande und limnischen Schluffe lassen sich wahrscheinlich stratigraphisch mit dem Offleben-Interstadial korrelieren. Eine sedimentologisch oder palynologisch belegbare Zweiteilung ist im Gegensatz zu Schöningen z. Zt. nicht möglich. Auf ein längeres Stadial sind die im Allertal weit verbreiteten warvitischen Beckenablagerungen zurückzuführen. Sie entsprechen vermutlich der im Tagebau Schöningen nicht näher untersuchten „Feinsandserie“, zufälligerweise auch in ihrer maximalen Mächtigkeit. Die hangenden, wahrscheinlich mit dem Esbeck-Interstadial zu parallelisierenden humosen Schluffe, Sande und Kalkmuddelagen sind leider nur in wenigen Bohrungen angetroffen wurden.

Während bei Schöningen eine mächtige Fließerde den Abschluß der Spätelster-zeitlichen Schichtenfolge bildet, kamen im Allertal in diesem Zeitraum vorwiegend Beckenbildungen zum Absatz. Ein zwar markanter, jedoch nur in einer Bohrung angetrof-

Abb. 5: Darstellung der Herkunft der Leitgeschiebe der Grobkiesfraktion von Elster-Grundmoränen.

a: Tagebau Schöningen, Elster-I-Geschiebemergel (TsCHEE 1991) b: Tagebau Schöningen, Elster-II-Geschiebemergel (ELSNER 1987) c: Tagebau Schöningen, Elster-II-Geschiebemergel (TSCHEE 1991)

Fig. 5: Diagram of origin of indicator stones in the coarse gravel fraction of tills of Elsterian age.

a: open-cast mine Schöningen, Elsterian-I-till (TsCHeE 1991)

b: open-cast mine Schöningen, Elsterian-II-till (ELSNER 1987)

c: open-cast mine Schöningen, Elsterian-II-till (TsCHEE 1991)

Abb. 6: Vergleichende Lage der TGZ von Elster-Grundmoränen.

( $1=$ Steinsohle und Geschiebelehmrest auf dem Elm, Look 1968; 2 = Geschiebemergel bei Velpke, Richter 1962; 3 = Geschiebemergel bei Velpke, MеRкт 1996, 4 = Tagebau Schöningen, Elster-I-Geschiebemergel, TscheE 1991; 5 = Tagebau Schöningen, Elster-II-Geschiebemergel, ELSNER 1987; 6 = Tagebau Schöningen, Elster-II-Geschiebemergel, TsCHEE 1991)

Fig. 6: Comparison of centres of indicator stone counts of tills of Elsterian age.

( 1 = lag deposit and rest of decalcified till on top of Elm ridge, LOOK 1968; 2 = till near Velpke, RicHTER 1962; 3 = till near Velpke, MerkT 1996, 4 = open-cast mine Schöningen, ElsterianI-till, TsCHEE 1991; 5 = open-cast mine Schöningen, Elsterian-II-till, ELSNER 1987; 6 = open-cast mine Schöningen, Elsterian-II-till, TscheE 1991) fener und deswegen nur unsicher stratigraphisch einstufbarer Boden läßt dort noch einmal wärmere Klimabedingungen vermuten.

\section{2 Überregionale Korrelation und Einstufung}

Mehr als im Detail problematisch gestaltet sich die Beantwortung der Frage, wie die im Untersuchungsraum beobachteten Elster-Eisvorstöße mit den häufig beobachteten zwei Vorstößen des ElsterInlandeises in Nord- und Mitteldeutschland zu korrelieren sind.

Bekanntermaßen liegen die umfangreichsten und detailliertesten Beobachtungen zum Elster-zeitlichen Geschehen aus Sachsen vor. Sie wurden u.a. zuletzt sehr ausführlich von EISSMANN \& LITT (1994) zusammengefaßt. Danach ist die untere Elster-Grundmoräne (sog. Zwickauer Phase) z. B. im Bitterfelder Raum (EIsSMann \& LitT 1994: 253254) durchschnittlich $2,5 \mathrm{~m}$ bzw. maximal $8 \mathrm{~m}$ mächtig, stets kalkhaltig $\left(8-12 \% \mathrm{CaCO}_{3}\right)$, von dunkel- bis schwarzolivgrauer Farbe und besitzt eine relativ homogene Struktur. Ihre mittlere Korngrößenzusammensetzung ist in Tabelle 1 wiedergegeben; Großgeschiebe sind nicht selten. Nur die untersten 1 bis $1,5 \mathrm{~m}$ der Grundmoräne enthalten zahlreiche Nester, Schlieren und Linsen aus Sand, während der massige obere Moränenteil als typischer Absetztill („lodgement till“) ausgebildet ist.

Teilweise über Zwischenmitteln des sog. „Miltitzer Intervalls" folgt die obere Elster-Grundmoräne (sog. Makranstädter Phase) als inhomogener Geschiebemergel $\left(1,5-3 \% \mathrm{CaCO}_{3}\right)$ von schwarzbraungrauer bis schwarzrotbrauner Farbe. Ihre mittlere Mächtigkeit beträgt nur $3 \mathrm{~m}$. Große Geschiebe sind selten (vgl. Tab. 1), dafür finden sich zahlreiche Tertiärkomponenten, v. a. Xylite und Schwefelkieskonkretionen. Typisch sind Scherflächen, Nester, Schlieren und Linsen aus Feinsand, Braunkohlebrocken und Xylitresten. Ähnliche Beobachtungen liegen aus dem Tagebau Gröbern bei Gräfenhainichen vor (EIsSMANN \& LITT 1994: 274-275; Wansa 1991, Hoffmann \& Meyer 1997), wo der obere Elster-Geschiebemergel jedoch wesentlich feinkörniger ausgebildet ist als der untere. Der obere Elster-Geschiebemergel ähnelt aufgrund seiner Feinkörnigkeit sehr dem zweiten Geschiebemergel von Schöningen (Tab. 1). Ein- 
Tab. 1: Mittlere Korngrößenzusammensetzungen von Elster-Grundmoränen in Nord- und Mitteldeutschland.

Tab. 1: Average grain sizes of tills of Elsterian age in northern and middle Germany.

\begin{tabular}{|c|c|c|c|c|c|c|c|}
\hline Bezeichnung / Lage (Quelle) & Ton & Schluff & Sand & Feinsand & Mittelsand & Grobsand & Kies \\
\hline $\begin{array}{c}\text { 2. Elster-Grundmoräne } \\
\text { Tagebau Schöningen (OLTMANNS 1993) }\end{array}$ & $8 \%$ & $54 \%$ & $35 \%$ & $18 \%$ & $13 \%$ & $4 \%$ & $3 \%$ \\
\hline $\begin{array}{c}\text { 1. Elster-Grundmoräne } \\
\text { Allertal (BGR unveröffentlicht) }\end{array}$ & $9 \%$ & $34 \%$ & $51 \%$ & $26 \%$ & $19 \%$ & $6 \%$ & $6 \%$ \\
\hline $\begin{array}{l}\text { 2. Elster-Grundmoräne } \\
\text { Allertal (BGR unveröffentlicht) }\end{array}$ & $11 \%$ & $33 \%$ & $51 \%$ & $26 \%$ & $19 \%$ & $6 \%$ & $5 \%$ \\
\hline $\begin{array}{c}\text { 1. Elster-Grundmoräne } \\
\text { Raum Bitterfeld (EISSMANN \& LITT 1994) }\end{array}$ & $10 \%$ & $27 \%$ & $58 \%$ & $29 \%$ & $22 \%$ & $7 \%$ & $5 \%$ \\
\hline $\begin{array}{c}\text { 2. Elster-Grundmoräne } \\
\text { Raum Bitterfeld (EISSMANN \& LITT 1994) }\end{array}$ & $7 \%$ & $34 \%$ & $54 \%$ & $24 \%$ & $23 \%$ & $7 \%$ & $5 \%$ \\
\hline $\begin{array}{c}\text { 1. Elster-Grundmoräne } \\
\text { Tagebau Gröbern (WANSA 1991) }\end{array}$ & $11 \%$ & $31 \%$ & $53 \%$ & $26 \%$ & $20 \%$ & $7 \%$ & $5 \%$ \\
\hline $\begin{array}{c}\text { 2. Elster-Grundmoräne } \\
\text { Tagebau Gröbern (WANSA 1991) }\end{array}$ & $17 \%$ & $53 \%$ & $28 \%$ & $21 \%$ & $5 \%$ & $2 \%$ & $2 \%$ \\
\hline $\begin{array}{c}\text { 1. Elster-Grundmoräne } \\
\text { Raum Gorleben (HEDERICH frdl. schriftl. Mitt.) }\end{array}$ & $21 \%$ & $28 \%$ & $47 \%$ & $20 \%$ & $21 \%$ & $6 \%$ & $4 \%$ \\
\hline $\begin{array}{c}\text { 2. Elster-Grundmoräne } \\
\text { Raum Gorleben („Geschiebesand“) (HEDERICH 1993) }\end{array}$ & $11 \%$ & $28 \%$ & $59 \%$ & $24 \%$ & $27 \%$ & $8 \%$ & $2 \%$ \\
\hline $\begin{array}{c}\text { jüngste Elster-Grundmoräne } \\
\text { Brüchau (,,Geschiebesand“) (HEDERICH 1993) }\end{array}$ & $7 \%$ & $33 \%$ & $59 \%$ & $33 \%$ & $22 \%$ & $4 \%$ & $1 \%$ \\
\hline $\begin{array}{l}\text { 1. Elster-Grundmoräne } \\
\text { Freißenbüttel N` Bremen (ScHWARZ frdl. schriftl. Mitt.) }\end{array}$ & $10 \%$ & $20 \%$ & $70 \%$ & $38 \%$ & $26 \%$ & $6 \%$ & $0 \%$ \\
\hline $\begin{array}{l}\text { 2. Elster-Grundmoräne } \\
\text { Freißenbüttel N` Bremen (,Geschiebesand“) } \\
\text { (SCHWARZ frdl. schriftl. Mitt.) }\end{array}$ & $9 \%$ & $6 \%$ & $85 \%$ & $68 \%$ & $14 \%$ & $3 \%$ & $0 \%$ \\
\hline
\end{tabular}


regelungsmessungen im unteren Elster-Geschiebemergel ergaben hier ein deutliches N-S-Maximum.

Wendet man sich dem nördlich anschließenden Bundesland Brandenburg zu (Lippstreu in BendA 1995), treten hier generell ebenfalls zwei ElsterGrundmoränen auf. Zahlreiche Rinnen und Wannen verhindern Aussagen, wie weit das Inlandeis zwischen dem 1. und dem 2. Eisvorstoß zurückgeschmolzen ist. Durch die große Tiefenlage der meist in Spülbohrungen innerhalb dieser Rinnen angetroffenen Elster-Geschiebemergel sind auch kaum Aussagen darüber möglich, wie diese Grundmoränen genau ausgebildet sind oder ob Oszillationen auftraten. Als gesichert gilt jedoch, daß der erste Elster-Eisvorstoß vergleichsweise schnell und ohne größere Stagnationen verlief. In Sachsen gehen Wolf \& AlEXOWSKY (1994: 198) von einem gleichmäßigen, unter extrem kalt-ariden Bedingungen jedoch relativ langsamen ersten Elster-Eisvorstoß aus.

Auch in Niedersachsen sind Grundmoränen von zwei Elster-Eisvorstößen vorhanden. Im Gegensatz zum mitteldeutschen Raum ist die oberste ElsterGrundmoräne jedoch häufig sehr sandig, als Geschiebesand mit sehr geringer Geschiebeführung ausgebildet (vgl. Tab. 1). Im Aufschluß nur schwer, in Kernbohrungen nur bei größter Sorgfalt und in Spülbohrungen auf keinen Fall erkennbar, ist das Auftreten dieses Geschiebesandes zumindest für den Raum Bremen und Gorleben (Meyer 2000) gesichert.

Möglicherweise hinterließ dieser letzte ElsterEisvorstoß jedoch nicht nur im nördlichen Niedersachsen den o.g. Geschiebesand mit geringer Geschiebeführung, sondern auch in Mecklenburg bei Hagenow (RÜHBERG et al. in BENDA 1995: 98) und in der Altmark bei Brüchau. Den hier in Kernbohrungen erteuften Elster-Geschiebesand parallelisierte Hederich (1994: 25) aufgrund seiner Korngrößenzusammensetzung (vgl. Tab. 1) und seines Kalkgehaltes (max. 5,3\%) mit dem Geschiebesand aus dem Raum Gorleben.

Die in Hamburg (EHLERs in BENDA 1995) und Schleswig-Holstein (STEPHAN in BENDA 1995) auftretenden zwei Elster-Grundmoränen sind sich dagegen sehr ähnlich und können weder aufgrund ihrer Korngrößenzusammensetzung noch ihrer Geschiebeführung voneinander unterschieden werden.

Jegliche überregionale Parallelisierungen von in verschiedenen Gebieten auftretenden Elster-Grundmoränen müssen $\mathrm{z}$. Zt. aufgrund der immer noch viel zu geringen Datenmengen spekulativ bleiben. Dies trifft auch zu, obwohl auf den ersten Anschein die lokal beobachteten zwei Grundmoränen meist leicht zwei Elster-Eisvorstößen zugeordnet werden können (vgl. Hoffmann \& Meyer 1997, Meyer 2000).

Vergleicht man aber z. B. die Elster-Grundmoränen des Untersuchungsgebietes mit den Beobachtungen aus Sachsen, gibt es v. a. Ähnlichkeiten zwischen der dortigen meist sehr homogenen Grundmoräne des ersten Vorstoßes und der ebenfalls sehr homogenen und geschiebereichen Grundmoräne des zweiten Vorstoßes im Helmstedter Raum. Andererseits kann aber wohl aufgrund seiner Feinkörnigkeit (s. Tab. 1) der mächtige Geschiebemergel von Schöningen (2. Eisvorstoß) mit dem zweiten ElsterGeschiebemergel aus dem Tagebau Gröbern korreliert werden.

Vor einer vorschnellen Korrelation von Grundmoränen nur aufgrund von ähnlichen Korngrößenverteilungen sei jedoch gewarnt. Tabelle 1 zeigt deutlich, wie stark die Korngrößenverteilungen, hier am Beispiel von Elster-Grundmoränen, regional wechseln können.

Oszillationen des Inlandeises sind bisher aus jedem Vereisungszyklus nur aus den jeweiligen Randbereichen der Eisbedeckung bekannt geworden. Überträgt man diese Beobachtung auf die Verhältnisse während des ersten Eisvorstoßes im Allertal (s. Abschnitt 5.1), müßte der zu dieser Zeit vorrückende Gletscher nicht unbedingt in seinem ersten Anlauf sofort bis nach Sachsen vorgestoßen sein. Denkbar wäre, daß nach Erreichen des Haldenslebener $\mathrm{Ab}$ bruchs, der die Altmark vom Mittelgebirgsraum trennt, erste Elster-Inlandeisvorstöße schnell an Kraft verloren haben. Vielleicht haben zu dieser Zeit nur einige Gletscherzungen das Untersuchungsgebiet erreicht; eine Hauptentwässerung könnte schon damals über das „Urstromtal“ des Großen Bruchs erfolgt sein.

Auch während der Eisabbauphase wird es regional noch zu erneuten Eisvorstößen gekommen sein 
(vgl. z. B. Wolf \& Alexowsky 1994: 198-199, EISSMANN 1994: 76), die dann z. B. im Allertal die jüngste, immerhin knapp $9 \mathrm{~m}$ mächtige Grundmoräne hinterlassen haben könnten.

Diese sehr knappe Schilderung von Untersuchungsergebnissen von Elster-Grundmoränen aus Nord- und Mitteldeutschland basiert auf den z. Zt. verfügbaren Daten. Weitere Untersuchungen sind unumgänglich, um in Zukunft sicherere Aussagen über das Verhalten des Elster-Inlandeises treffen zu können.

\section{Danksagung}

Der Autor möchte dem Bundesamt für Strahlenschutz für die Erlaubnis zur Veröffentlichung von Daten aus dem Programm „Deckgebirgsuntersuchung ERA Morsleben“ danken, die jedoch erst nach Beendigung aller Deckgebirgsuntersuchungen und Vorlage aller Abschlußberichte wirksam wurde. Den Gutachtern von E \& G sei für ihre konstruktiven Vorschläge und der zum Teil überaus positiven Kritik gedankt.

Herr Prof. K.-D. MEYER, NLfB Hannover, führte freundlicherweise zahlreiche Geschiebezählungen nach der TGZ-Methode aus und ermöglichte die zeichnerische Umsetzung der Einzelergebnisse. Für Diskussionsbeiträge bin ich ihm, Prof. Dr. L. EIssmann, K. Hoffmann, beide Universität Leipzig, Drs. S. Wansa, W. Knoth und B. von Poblozki sowie L. StOtTmeister, alle LfGB Sachsen-Anhalt, Halle, L. Lippstreu, LGRB, Kleinmachnow, L. Wolf, SLUG, Freiberg, Dr. habil. J. Ehlers, Umweltbehörde Hamburg, Dr. H.-J. Stephan, LfNU Schleswig-Holstein, Kiel, Dr. F. M. vaN DER Wateren, Rijks Geologische Dienst, Haarlem, Dr. J. J. M. VAN DER MeER, Universiteit van Amsterdam, H. Ziermann, ehemals BGR-Außenstelle Berlin sowie N. HEDERICH und R. ZwIrner, beide Golder Associates GmbH, Celle, dankbar. H. ZIERmanN, N. Hederich sowie Dr. C. Schwarz, NLfB Hannover, stellten bereitwillig Analysen von Korngrößenverteilungen zur Verfügung.

Frau A. Hochmuth, Salzwedel, Frau A. Heyer, Volkmarshausen, sowie Herr S. LeIFeLD, Lienburg, übernahmen in bewährter Weise die Reinzeichnung der Abbildungen.

\section{Schriftenverzeichnis}

Amler, M. R. W. \& Elsner, H. (1988): Ptilodictya lanceolata aus obersilurischen Geschieben von Schöningen/Ost-Niedersachsen. - Geschiebekunde aktuell, 4(3): 69-80, 6 Abb., 1 Tab; Hamburg.

Bartholomäus, W. A. (1987): Weitere Funde der gelbbraunen Kieselkreide. - Der Geschiebesammler, 21(1): 43-46, 1 Abb; Hamburg.

Bartholomäus, W. A. \& Tschee, W. (1993): Ein unterkambrisches Geschiebeproblematikum von Schöningen/Ostniedersachsen. - Geschiebekunde aktuell, 9(1): 7-8, 2 Abb; Hamburg.

Bartholomäus, W. A. \& Elsner, H. (1995): Quartär bei Helmstedt: Über den derzeitigen quartärgeologischen Aufschlußstand im Braunkohlen-Tagebau Schöningen-Südfeld. - Der Geschiebesammler, 28(2): 63-72, $1 \mathrm{Abb}$; Wankendorf.

BENDA, L. (1995): Das Quartär Deutschlands. - herausgegeben von L. BENDA im Auftrag der Deutschen Quartärvereinigung: 408 S., 95 Abb., 30 Tab.; Berlin-Stuttgart (Borntraeger).

BGR (1993): Projekt Morsleben. Grundlagen zur hydrogeologischen Modellbildung. - Zwischenbericht 1993 der Bundesanstalt für Geowissenschaften und Rohstoffe, Außenstelle Berlin, für das Bundesamt für Strahlenschutz, BGR-Archiv-Nr. (Hannover): 111002: 180 S., 17 Tab., 19 Abb., 53 Anl.; Berlin, Hannover (unveröffentlicht).

Eissmann, L. (1994): Grundzüge der Quartärgeologie (Sachsen, Sachsen-Anhalt, Südbrandenburg, Thüringen). - In Eissmann, L. \& LitT, T. (Hrsg.): Das Quartär Mitteldeutschlands. Ein Leitfaden und Exkursionsführer. Mit einer Übersicht über das Präquartär des Saale-Elbe-Gebietes. - Altenbg. nat. wiss. Forsch., 7: 55-135, 35 Abb., 5 Tab., 12 Taf.; Altenburg.

Eissmann, L. \& LitT, T. (1994): Das Quartär Mitteldeutschlands. Ein Leitfaden und Exkursionsführer. Mit einer Übersicht über das Präquartär des SaaleElbe-Gebietes. - Altenbg. nat. wiss. Forsch., 7: 458 S., 174 Abb., 46 Taf., 22 Tab.; Altenburg.

EIssmann, L. \& LitT, T. (1994): Klassische Quartärfolge Mitteldeutschlands von der Elstereiszeit bis zum Holozän unter besonderer Berücksichtigung der Stratigraphie, Paläoökologie und Vorgeschichte (Exkursion B1). - In: Eissmann, L. \& LitT, T. (Hrsg.): Das Quartär Mitteldeutschlands. Ein Leitfaden und Exkursionsführer. Mit einer Übersicht über das Präquartär des Saale-Elbe-Gebietes. - Altenbg. nat. wiss. Forsch., 7: 250-236, 43 Abb., 6 Tab., 13 Taf.; Altenburg.

ELSNER, H. (1987): Das Quartär im Tagebau Schöningen der Braunschweigischen Kohlenbergwerke AG, Helmstedt. - Diplomarbeit am Fachbereich Erdwis- 
senschaften der Universität Hannover: 126 S.; Hannover (unveröffentlicht).

FEeser, V. (1986): Geomechanisches Konzept zur Gefügeentwicklung und Spannungsgeschichte glazialtektonisch überprägter Tone. - Braunschweiger geol.-paläont. Diss., 4: 127 S.; Braunschweig.

Feldmann, L., Groetzner, J.-P. \& Weymann, H.-J. (2001): Zur pleistozänen Geschichte des „Großen Bruch" im Nördlichen Harzvorland. - Geol. Beitr. Hannover, 2: 127-137, 4 Abb., 1 Tab.; Hannover.

Hartmann, T. (1988): Elster- bis Saale-zeitliche Sedimente im Tagebau Schöningen der Braunschweigischen Kohlen-Bergwerke AG, Helmstedt. - Diplomarbeit am Fachbereich Erdwissenschaften der Universität Hannover: 153 S.; Hannover (unveröffentlicht).

Hederich, N. (1993): Die pleistozäne Schichtfolge im Untergrund und der Umgebung der Sonderabfalldeponie Brüchau, Kreis Gardelegen (Altmark). - Diplomarbeit am Fachbereich Geowissenschaften der Universität Hannover: 75 S.; Hannover (unveröffentlicht).

Hoffmann, K. \& Meyer, K.-D. (1997): Leitgeschiebezählungen von elster- und saalekaltzeitlichen $\mathrm{Ab}$ lagerungen aus Sachsen, Sachsen-Anhalt und dem östlichen Niedersachsen. - Leipziger Geowissenschaften, 5: 115 - 128, 7 Abb., 3 Tab.; Leipzig.

LENHART, R. (1989): Schichtlagerung und Zusammensetzung Elster- bis Saale-zeitlicher Sedimente im Baufeld Esbeck, Tagebau Schöningen der Braunschweigischen Kohlen-Bergwerke AG, Helmstedt. Diplomarbeit am Fachbereich Erdwissenschaften der Universität Hannover: 125 S.; 52 Abb.; Hannover (unveröffentlicht).

Lietzow, A. \& Ritzkowski, S. (1996): Bergbau und die tertiäre Schichtenfolge. - In: Exkursion A 1: Braunschweigische Kohlenbergwerke AG, Tagebau Schöningen. - 63. Tagung der Arbeitsgemeinschaft Nordwestdeutscher Geologen vom 28. bis 31. Mai 1996 in Helmstedt. Kurzfassung der Vorträge und Exkursionsführer: 30-35, 2 Abb., 1 Tab.; NLfB, Hannover.

Look, E.-R. (1968): Geologisch-stratigraphische Untersuchungen in Sedimenten der Elster- und SaaleEiszeit (Pleistozän) am Elm, östlich Braunschweig. Mitt. geol. Inst. Techn. Hochschule Hannover, 6: 108 S., 18 Abb., 4 Tab., 27 Taf.; Hannover.

LÜtTIG, G. (1958): Methodische Fragen der Geschiebeforschung. - Geol. Jb., 75: 361-418; Hannover.

Merkт, J. (1996): Gletscherschrammen in einem aufgelassenen Steinbruch bei Velpke. - In: Exkursion A 2: Gebiet der Blätter 3431 Parsau und 3531 Oebisfelde der GK 25. - 63. Tagung der Arbeitsgemeinschaft Nordwestdeutscher Geologen vom 28. bis 31. Mai 1996 in Helmstedt. Kurzfassung der Vorträge und Exkursionsführer: S. 42, 1 Abb.; NLfB, Hannover. MEYER, K.-D. (2000): Geschiebekundlich-stratigraphi- sche Untersuchungen im Hannoverschen Wendland (Niedersachsen). - Brandenburgische Geowiss. Beitr., 7: 115 -126, 4 Abb., 2 Tab., 1 Taf.; Kleinmachnow.

Oltmanns, E. (1993): Die Wasserhaltung im Tagebau Schöningen (Braunschweigische Kohlenbergwerke AG, Helmstedt) unter besonderer Berücksichtigung schwer entwässerbarer Schichten. - Diplomarbeit am Institut für Geowissenschaften der TU Braunschweig: 86 S.; Braunschweig (unveröffentlicht).

Richter, K. (1961): Das Alter der Gletscherschrammen von Velpke. - Eiszeitalter und Gegenwart, 12: 125130, 3 Abb.; Öhringen/Württemberg.

Richter, K. (1962): Geschiebekundliche Gliederung der Elster-Eiszeit in Niedersachsen. - Mitt. Geol. Staatsinst. Hamburg, 31: 309-343, 2 Tab., 4 Abb.; Hamburg.

SChmierer, T. (1913): Über fossilführende Interglazialablagerungen bei Oschersleben und Ummendorf (Prov. Sachsen) und über die Gliederung des Magdeburg-Braunschweigischen Diluviums im allgemeinen. - Jb. preuß. geol. L.-A., 33, Tl. 2: 400-417, 1 Abb; Berlin.

Tschee, W. (1991): Die pleistozäne Schichtfolge im Tagebau Schöningen Baufeld Esbeck der Braunschweigischen Kohlen-Bergwerke AG, Helmstedt. Diplomarbeit am Fachbereich Erdwissenschaften der Universität Hannover: 75 S.; Hannover (unveröffentlicht).

Urban, B; Thieme, H. \& Elsner, H. (1988): Biostratigraphische, quartärgeologische und urgeschichtliche Befunde aus dem Tagebau „Schöningen“, Ldkr. Helmstedt. - Z. dt. geol. Ges., 139: 123-154, 9 Abb., 1 Taf.; Hannover.

Urban, B.; Lenhart, R.; Mania, D. \& Albrecht, B. (1991): Mittelpleistozän im Tagebau Schöningen; Ldkr. Helmstedt. - Z. dt. geol. Ges., 142: 351-372, 6 Abb., 2 Tab., 1 Taf.; Hannover.

VAN DER MEer, J. J. M. (1994): Micromorphology. - In: Menzies, J. (ed.): Past glacial environments. Sediments, forms and techniques., 2: 335 - 355, 28 plates; Butterworth \& Heinemann Ltd., Oxford.

Wagenbreth, O. (1970): Beobachtungen und Probleme im Quartär des Salzsattels von Staßfurt-EgelnOschersleben und seiner Randsenken. - Geologie, 9: 412-433, 13 Abb., 4 Tab.; Berlin.

WaHnsChafFE, F. (1880): Über Gletschererscheinungen bei Velpke und Danndorf. - Z. dt. geol. Ges., 32: 774-798; Berlin.

WANSA, S. (1991): Lithologie und Stratigraphie der Tills bei Gräfenhainichen. - Mauritiana, 13, 1/2: 189211, 11 Abb., 4 Tab.; Altenburg.

Wiegers, F. (1932): Das Alter des Diluvium in der Gegend zwischen Oschersleben-Bode und Staßfurt und der Verlauf der diluvialen Bode-Terrasse. - Jb. preuß. geol. L.-A., 52: 447-456; Berlin. 
Wolf, L. \& Alexowsky, W. (1994): Fluviatile und glaziäre Ablagerungen am äußersten Rand der Elster- und Saale-Vereisung; die spättertiäre und quartäre Geschichte des sächsischen Elbgebietes (Exkursion A 2). - In: Eissmann, L. \& Litt, T. (Hrsg.): Das Quartär Mitteldeutschlands. Ein Leitfaden und Exkursionsführer. Mit einer Übersicht über das Präquartär des Saale-Elbe-Gebietes.- Altenbg. nat. wiss. Forsch., 7: 190-235, 18 Abb., 5 Tab., 1 Taf.; Altenburg. 